TRANSACTIONS OF THE

AMERICAN MATHEMATICAL SOCIETY

Volume 188, Issue 2, 1974

\title{
SYMMETRIC NORM IDEALS AND RELATIVE CONJUGATE IDEALS
}

BY

\author{
NORBERTO SALINAS( $($ )
}

ABSTRACT. In this paper some aspects of the algebraic structure of the ring of all bounded linear operators on an infinite dimensional separable complex Hilbert space are discussed. In particular, a comparison criterion for maximal and minimal norm ideals is established. Also, a general notion of the conjugate of an ideal relative to another ideal is studied and some questions concerning joins and intersections of ideals are solved.

1. Introduction. Throughout this paper $\mathcal{L}$ will denote the ring of all (bounded linear) operators on a fixed separable infinite dimensional complex Hilbert space. The term ideal of $\mathcal{L}$ will mean a two sided ideal of the ring $\mathcal{L}$. The maximal ideal of $\mathcal{L}$, i.e. the set of all compact operators, will be denoted by $\mathcal{C}$, and $\mathfrak{F}$ will denote the set of all finite rank operators, which is the minimal ideal of $\mathfrak{L}$.

In the present paper we investigate the notion of the conjugate ideal of an ideal relative to another ideal. For a given ideal $\mathcal{K}$ of $\mathscr{L}$, the conjugate ideal of an ideal $\mathscr{I}$ of $\mathscr{L}$ relative to $\mathcal{K}$, or simply the $\mathcal{K}$-conjugate ideal of $\mathscr{G}$, is the set of all operators $T$ in $\mathfrak{L}$ such that $T S \in \mathcal{K}$, for every $S \in \mathcal{G}$. The $\mathcal{K}$-conjugate of $g$ can also be characterized as the largest of those ideals $g$ such that $I 9 \subseteq \mathcal{K}$ (\$3). If $\mathcal{K}$ is the trace class $\mathcal{C}_{1}$, the $\mathcal{K}$-conjugate of an ideal $\mathcal{G}$ coincides with the Köthe-dual of $\mathcal{G}[4, \S 5]$. Furthermore, if $\mathcal{G}$ is a minimal norm ideal, then the $C_{1}$-conjugate of $g$ is the topological dual of $\mathcal{G}[12$, Chapter 5$]$.

Our main purpose in this note is to present some of the facts of the theory of conjugate ideals in a more transparent and elementary fashion via the above more general and algebraic definition of conjugate of an ideal. For instance, if $\mathcal{K}$ is a proper ideal we shall see $\left(\S_{3}\right)$ that with our definition of $K$-conjugate it is very simple to prove that if there exists a self $\mathcal{K}$-conjugate ideal, then it must coincide with the square root of $\mathcal{K}$ (where $\mathcal{K}^{1 / 2}$ is defined in the natural way $[11, \S 2]$ ).

On the other hand, some of our results also lead to new theorems of the standard theory. In $\$ 4$ we discuss the case in which $\mathcal{K}$ is a symmetric norm ideal, and to this end we establish a comparison criterion for maximal and minimal norm

Received by the editors June 23, 1972 and, in revised form, April 17, 1973. AMS (MOS) subject classifications (1970). Primary 47B05, 47K05; Secondary 47B10, 46A45.

(1) Supported in part by National Science Foundation grant GP-7952X3. 
ideals in $\S 2$. We show $(\S 4)$ that if $p>1, q>1,1 / p+1 / q=1$, and $K$ is a maximal norm ideal, then $K^{1 / p}$ and $K^{1 / q}$ are $K$-conjugates one of another, and a Hölder type inequality holds for a pair of operators $S \in K^{1 / p}, T \in K^{1 / q}$. In particular $\mathcal{K}^{1 / p K^{1 / q}=K}$. Even for the classical case $\left(K=\mathcal{C}_{1}\right)$ this result explains the rather mysterious relation $1 / p+1 / q=1$ that the numbers $p$ and $q$ must satisfy.

Following [1] a nonnegative convex function $f$ defined on the nonnegative line will be called admissible whenever $f(\xi)>0$ if and only if $\xi>0$. In $\$ 5$ we introduce the ideals $\mathcal{S}(f ; \mathcal{K})$ and $\mathscr{D}(f ; \mathcal{K})$ as in [10], where $\mathcal{K}$ is an ideal of $\mathcal{L}$ and $f$ is an admissible function. These ideals may be defined as follows:

$$
\begin{aligned}
& \mathcal{S}(f ; \mathcal{K})=\left\{T \in \mathscr{L}: f\left(\alpha \sqrt{T^{*} T}\right) \in \mathcal{K}, \text { for some } a>0\right\}, \\
& \mathcal{D}(f ; \mathcal{K})=\left\{T \in \mathscr{L}: f\left(\alpha \sqrt{\left.T^{*} T\right)} \in \mathcal{K}, \text { for all } a>0\right\} .\right.
\end{aligned}
$$

We prove that if $\mathcal{K}$ is a maximal norm ideal and $f$ is a convex admissible function, then $\mathcal{S}(f ; \mathcal{K})$ is a maximal norm ideal and we show that the corresponding minimal norm ideal is given by $\mathscr{D}\left(f ; K^{0}\right)$, where $\mathcal{K}^{0}$ is the minimal norm ideal corresponding to $K$. $\$ 6$ is devoted to proving that if $f$ and $g$ are convex admissible functions conjugate in the sense of Young, then $\mathcal{S}(f ; \mathcal{K})$ and $\delta(g ; \mathcal{K})$ are $\mathcal{K}$-conjugates one of another, where $\mathcal{K}$ is a maximal norm ideal. Furthermore, it is shown that $\mathcal{S}(g ; \mathcal{K})$ is the $\mathcal{K}$-conjugate and the $\mathcal{K}^{0}$-conjugate of $\mathscr{L}\left(f ; K^{0}\right)$. If $\mathcal{K}=\mathcal{C}_{1}$ then the ideals $\mathcal{S}(f ; \mathcal{K})$ and $\mathscr{L}(f ; \mathcal{K})$ become the ideals $\mathcal{S}(f)$ and $\mathscr{I}(f)$ defined in [1]. Thus, the above results imply, in particular, that $\mathcal{S}(f)$ is a maximal norm ideal and $\mathscr{D}(\rho)$ is the corresponding minimal norm ideal. Also, if $g$ is the conjugate of $f$ in the sense of Young, then $S(f)$ and $S(g)$ are Köthe-duals one of each other, and $S(g)$ is the Kothe and topological dual of $\mathscr{T}(f)$. Finally, in $\$ 7$ we make some remarks concerning joins and intersections of ideals. As a by-product of this discussion, we prove that the join of a countable family of norm ideals properly contained in $\mathcal{C}$ is strictly smaller than $\mathcal{C}$. This result answers, in the negative, for the case of norm ideals, a question raised in $[1, \$ 6]$. Also, we prove that the union of an increasing countable family of ideals strictly contained in $\mathcal{C}$ is properly contained in $\mathcal{C}$, reducing the above mentioned problem of [1] to the question of whether or not $\mathcal{C}$ can be expressed as the join of two strictly smaller ideals.

2. Maximal and minimal norm ideals. We begin our considerations by reviewing some known results from the theory of ideals of $\mathfrak{L}[5$, Chapter 3$]$.

One of the basic facts we shall repeatedly use in the sequel is a theorem of Calkin $[2, \S 1]$ which establishes a lattice isomorphism between the set of ideals of $\mathcal{L}$ and the set of characteristic sets (see also [4]). In order to state this result we need to introduce some notation.

Let $\mathfrak{R}$ be the set of all nonincreasing sequences of nonnegative real numbers.

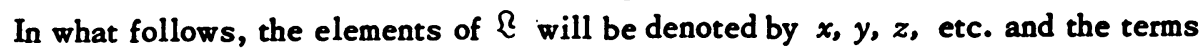


of the sequence $x$ by $x_{n}, n=1,2, \ldots$ Given $x, y \in\{$ and a nonnegative real number $a$ we denote by $x+y$ and $x y$ the elementwise sum and product of the sequences $x$ and $y$, and by $a x$ the sequence whose terms are $a x$.

A characteristic set $\mathfrak{I}$ is a subset of $\mathfrak{Z}$ that has the following properties:

(I) If $x \in \Im$, then the sequence $\left\{x_{1}, x_{1}, x_{2}, x_{2}, x_{3}, x_{3}, \ldots\right\}$ is in $\Im$

(II) If $x, y \in \mathfrak{F}$, then $x+y \in \mathfrak{F}$.

(III) If $x \in \mathfrak{J}, y \in\left\{\right.$, and $y_{n} \leq x_{n}, n=1,2, \ldots$, then $y \in \Im$.

Following the standard notation we shall denote by $s(T)$ the sequence of singular numbers (also called approximating numbers) of the operator $T \in \mathscr{L}$. If $\mathcal{F}_{n}$ denotes the set of all operators of rank at most $n$, then

$$
s_{n}(T)=\inf _{X \in \mathcal{F}_{n-1}}\|T-X\|, \quad n=1,2, \ldots \ldots
$$

If $T$ is a compact operator, then $s(T)$ is the sequence of eigenvalues of the operator $\sqrt{T^{*} T}$, counted according to multiplicity and arranged in decreasing order of magnitude.

The above mentioned Calkin's theorem states that if $\mathcal{I}$ is an ideal of $\mathcal{L}$, then the set $\Lambda(\mathcal{G})$ of all sequences of the form $s(T), T \in \mathcal{G}$ is a characteristic set and the correspondence $\mathcal{I} \leftrightarrow \Lambda(\mathcal{G})$ is a lattice isomorphism. Thus, $\Lambda(\mathcal{C})$ is the set of

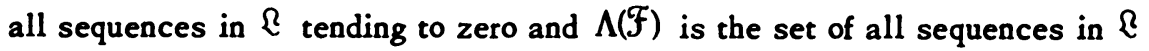
having only finitely many terms different from zero. Since the characteristic set $\Lambda(\mathcal{F})$ will be frequently used throughout this paper we shall write $\mathfrak{F}$ in place of $\Lambda(\mathcal{F})$. If $\mathcal{C}_{p}$ denotes the $p$-Schatten norm ideals, $p \geq 1$ [12], then we also have $\Lambda\left(\mathcal{C}_{p}\right)=\left\{x \in \in:\left\{: \Sigma_{n=1}^{\infty} x_{n}^{p}<\infty\right\}\right.$.

Following [12] we shall say that an ideal $\mathscr{G}$ of $\mathfrak{L}$ is a norm ideal if there exists a norm $\|\cdot\|_{g}$ on $\mathcal{G}$ satisfying the following conditions:

(a) $\left(\mathcal{G},\|\cdot\|_{g}\right)$ is a Banach space.

(b) $\|S T R\|_{g} \leq\|S\|\|R\|\|T\|_{g}$, for every $R, S \in \mathscr{L}, T \in \mathcal{G}$.

(c) $\|T\|_{g}=\|T\|$, for every operator $T$ of rank one.

Let $\mathcal{P}$ denote the directed set of all finite rank (orthogonal) projections in $\mathcal{L}$. It can be easily proved that if $T \in \mathcal{G}$, then $\left\{\|P T\|_{g}, P \in \mathcal{P}\right\}$ is an increasing net of nonnegative real numbers directed by the set $\mathscr{P}$. If $\left(g^{\prime},\|\cdot\|_{g}\right)$ is a norm ideal, and, in addition to $(\mathrm{a}),(\mathrm{b})$ and $(\mathrm{c}),\|\cdot\|_{\mathrm{g}}$ satisfies:

(d) $\lim _{P \in g}\|P T\|_{g}=\|T\|_{g}$, for every $T \in \mathscr{I}$, then $\left(\mathcal{G},\|\cdot\|_{g}\right)$ is called a symmetric norm ideal $[9, \S 7]$. If $\left(\mathcal{g},\|\cdot\|_{g}\right)$ is a norm ideal, then the closure of $\mathcal{F}$ in the topology of $\mathscr{G}$ is the smallest norm ideal with norm $\|\cdot\|_{g}$. Following [12] we shall say that $\left(\mathcal{G},\|\cdot\|_{g}\right)$ is a minimal norm ideal whenever $\mathcal{I}$ coincides with the closure of $\mathcal{F}$ in the topology of $\mathcal{G}$. We shall see below that minimal norm ideals are symmetric norm ideals.

If $\left(\mathcal{G},\|\cdot\|_{\mathcal{G}}\right)$ is a norm ideal and $T \in \mathcal{G}$, it readily follows that $\|T\|_{\mathcal{G}}$ depends 
only on the sequence of singular numbers of $T$. Let $\Phi$ be the nonnegative function defined on $\mathfrak{F}$ by the identity

$$
\Phi[s(T)]=\|T\|_{\mathbf{j}}, \quad T \in \mathfrak{F} \text {. }
$$

Then $\Phi$ satisfies the following properties, for every $x, y \in \mathfrak{F}$.

(i) If $x_{1}>0, \Phi(x)>0$.

(ii) $\Phi(x+y) \leq \Phi(x)+\Phi(y)$.

(iii) $\Phi(\alpha x)=\alpha \Phi(x)$, for every $a \geq 0$.

(iv) If $v=\{1,0,0, \ldots\}$, then $\Phi(v)=1$.

(v) If $\sum_{n=1}^{k} x_{n} \leq \Sigma_{n=1}^{k} y_{n}, k=1,2, \cdots$, then $\Phi(x) \leq \Phi(y)$.

A nonnegative function $\Phi$ on $\mathfrak{F}$ that satisfies the properties $(i)-(v)$ will be called a symmetric norming function, and the set of these functions will be denoted by $\pi$. It follows directly from ( $v$ ) that if $\Phi \in \boldsymbol{r}$, then $\Phi$ is a nondecreasing function, that is $\Phi(x) \leq \Phi(y)$, whenever $x_{n} \leq y_{n}, n=1,2, \ldots$. Therefore, if $\sigma_{k}(x)$ denotes the $k$ th section of the sequence $x \in \mathbb{R}$ (i.e. $\left.\sigma_{k}(x)=\left\{x_{1}, x_{2}, \ldots, x_{k}, 0,0, \ldots\right\}\right)$, then $\left\{\Phi\left[\sigma_{k}(x)\right]\right\}$ constitutes a nondecreasing sequence of nonnegative real numbers, for each fixed $x \in \mathbb{R}$. It follows that the set

$$
\tilde{G}_{\Phi}=\left\{x \in \mathfrak{R}: \lim _{k \rightarrow \infty} \Phi\left[\sigma_{k}(x)\right]<\infty\right\}
$$

is a characteristic set and is the natural domain of definition of the function $\Phi$. It can be easily checked that $\Phi$, as a function on $\mathcal{G}_{\phi}$, satisfies the properties (i)-(v) described above and

(vi) $\lim _{k \rightarrow \infty} \Phi\left[\sigma_{k}(x)\right]=\Phi(x)$, for every $x \in \mathcal{G}_{\Phi}$. If $r_{k}(x)$ denotes the $k$ th tail of $x \in\left\{\right.$ (i.e. $r_{k}(x)=\left\{x_{1+k}, x_{2+k}, \ldots\right\}$ ), then the set

$$
\mathcal{G}_{\Phi}^{0}=\left\{x \in \mathcal{G}_{\Phi}: \lim _{k \rightarrow \infty} \Phi\left[r_{k}(x)\right]=0\right\}
$$

is a characteristic set which is intimately related to the function $\Phi$.

With the following theorem, we complete the review of the known results we shall need concerning symmetric norm ideals. Its proof is essentially contained in [5, Chapter 3].

Theorem 2.1. Let $\left(\mathcal{G},\|\cdot\|_{g}\right)$ be a norm ideal and let $\Phi$ be the symmetric norming function induced by $\|\cdot\|_{g}$. Furtbermore, let $\delta_{\$}$ be the ideal of $\mathcal{L}$ defined by $\Lambda\left(\delta_{\phi}\right)=\mathcal{G}_{\phi}$. Then we have:

(a) $\mathcal{S}_{\phi}=\left\{T \in \mathscr{L}: \lim _{P \in \Phi}\|P T\|_{g}<\infty\right\}$.

(b) $\mathcal{S}_{\phi}$ is a norm ideal with the norm $\|T\|_{\Phi}=\lim _{P \in \Phi}\|P T\|_{g}=$ $\lim _{k \rightarrow \infty}\left(\Phi\left(\sigma_{k}[s(T)]\right), T \in \delta_{\phi}\right.$.

(c) $g \subseteq \delta_{\Phi}$ and $\|T\|_{\Phi} \leq\|T\|_{g}, T \in G$.

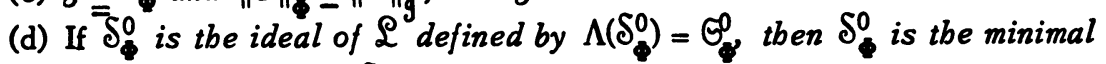
norm ideal corresponding to $\mathcal{S}_{\text {. }}$. 
Remark 2.2. Let $\left(g,\|\cdot\|_{g}\right)$ be a norm ideal, and let $\Phi$ be the symmetric norming function induced by $\|\cdot\|_{\mathrm{g}}$. From Theorem 2.1 we conclude that $\delta_{\phi}$ is the largest of those norm ideals whose minimal norm ideals coincide with $\delta_{\phi}^{0}$. In view of the preceding observation a norm ideal $\left(\mathcal{G},\|\cdot\|_{\mathcal{g}}\right)$ satisfying $\mathcal{I}=\{T \in \mathscr{L}$ : $\left.\lim _{P \in \Phi}\|P T\|_{g}<\infty\right\}$ will be called maximal norm ideal. It follows from Theorem 2.1 that a norm ideal is a maximal (minimal, resp.) norm ideal if and only if it is of the form $\delta_{\Phi}\left(\aleph_{\Phi}^{0}\right.$, resp. $)$, for some $\Phi \in \mathcal{H}$. In the following theorem we establish a comparison test for symmetric norming functions which can also be used to compare maximal and minimal norm ideals.

Theorem 2.3. Let $\Phi, \Psi \in \Re$. Then the following conditions are equivalent:
(a) $\delta_{\vartheta} \subseteq \delta_{\phi}$.
(b) $\mathcal{S}_{\Psi}^{0} \subseteq \mathcal{S}_{\odot}^{0}$.
(c) $\delta_{\Psi}^{0} \subseteq \delta_{\phi}$
(d) There exists $\gamma>0$ such that $\Phi(x) \leq \gamma \Psi(x)$, for every $x \in \mathfrak{F}$.
(e) $\sup _{x \in \delta, x_{1} \neq 0}[\Phi(x) / \Psi(x)]<\infty$.

Proof. It is clear that (a) $\Rightarrow$ (c), (b) $\Rightarrow(c),(d) \Rightarrow(a),(d) \Rightarrow$ (b) and (d) $\Rightarrow$ (e). Since symmetric norming functions are monotone we see that for every $T \epsilon \mathcal{S}_{\phi}$,

$$
\|T\|=s_{1}(T)=s_{1}(T) \Phi(\{1,0,0, \ldots\})=\Phi\left(\left\{s_{1}(T), 0,0, \ldots\right\}\right) \leq \Phi[s(T)]=\|T\|_{\Phi} \cdot
$$

Similarly, $\|T\| \leq\|T\|_{\Downarrow}$, for every $T \in \int_{*}^{0}$. Therefore, the canonical inclusion maps from $\mathcal{S}_{\Phi}$ into $\mathcal{L}$ and from $\mathcal{S}_{\Psi}^{0}$ into $\mathcal{L}$ are continuous. From the closed graph theorem it follows that the canonical inclusion map from $\delta_{\Psi}^{0}$ into $\mathfrak{S}_{\Phi}$ is also continuous. Whence, there exists $\gamma>0$ such that $\|T\|_{\Phi} \leq \gamma\|T\|_{\Psi}$, for every $T \in \mathcal{S}_{\Psi}^{0}$. Now, for every $x \in \mathfrak{F}$ we choose $T \in \mathcal{F}$ such that $x=s(T)$. We deduce that $\Phi(x)=\Phi[s(T)]=$ $\|T\|_{\Phi} \leq \gamma\|T\|_{\Psi}=\gamma \Psi[s(T)]=\gamma \Psi(x)$ and part (d) follows.

Corollary 2.4. Let $\Phi, \Psi$ be in $\pi$. If $\delta_{\Phi}=\delta_{\Psi}^{0}$ then $\delta_{\Psi}=\delta_{\Psi}^{0}$ and $\delta_{\Phi}=\delta_{\Phi}^{0}$. In particular $\mathcal{S}_{\Phi} \subseteq \mathcal{C}$ if and only if $\mathcal{S}_{\Phi} \subseteq \mathcal{C}$.

Remark 2.5. In view of Theorem 2.3 it is natural to define the following order relation and equivalence relation on $\pi$ : If $\Phi, \Psi \in \pi$, then $\Phi \ll \Psi$ if and only if any of the statements of Theorem 2.3 holds; also, $\Phi \approx \Psi$ if and only if $\Phi \prec<\Psi$ and

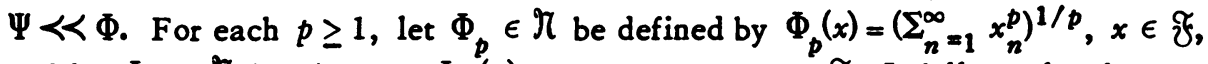
and let $\Phi_{\infty} \in \Re$ be given by $\Phi_{\infty}(x)=\sup _{n} x_{n}=x_{1}, x \in \mathcal{F}$. It follows that for every $\Phi \in \Re$, and every $x \in \mathfrak{F}$ we have $\Phi_{\infty}(x) \leq \Phi(x) \leq \Phi_{1}(x)$. For this reason, $\Phi_{1}$ and $\Phi_{\infty}$ are called the maximal and minimal norming functions, respectively. In [5, Chapter III, $\left.\S_{3}\right]$ it is shown that $\Phi \approx \Phi_{\infty}$ if and only if $\sup _{k} \Phi\left[\sigma_{k}(u)\right]<\infty$, and $\Phi \approx \Phi_{1}$ if and only if $\inf _{k} k^{-1} \Phi\left[\sigma_{k}(u)\right]>0$, where $u$ denotes the constant sequence $u_{n}=1, n=1,2, \ldots$. Observe that $\mathcal{S}_{\Phi_{\infty}}=\mathcal{L}, \mathcal{S}_{\Phi_{\infty}}=\mathcal{C}$, and 
$\delta_{\Phi_{1}}=\delta_{\Phi_{1}}^{0}=\mathcal{C}_{1}$. A function $\Phi \in \boldsymbol{H}$ is said to be mono-normalizing whenever $\delta_{\Phi}=$ $\oint_{\Phi}^{0}$. From Corollary 2.4 it follows that if $\Phi$ is a mono-normalizing function and $\Psi \approx \Phi$, then $\Psi$ enjoys the same property. Let us denote by $\widetilde{\Phi}$ the equivalence class of $\Phi$. From Theorem 2.3 we conclude that the mappings $\tilde{\Phi} \rightarrow \delta_{\phi}$, and $\widetilde{\Phi} \rightarrow \delta_{\phi}^{0}$, from the set of equivalence classes of $\pi$ to the set of maximal norm ideals and the set of minimal norm ideals, respectively, are bijective and order reversing. Moreover, it follows from Corollary 2.4 that they only coincide in the set of equivalence classes of mono-normalizing functions. In Remark 4.7(d) we shall show that the set of maximal norm ideals and the set of minimal norm ideals are lattices as well as the set of equivalence classes of $\pi$, and we shall see that the mappings mentioned above also preserve the corresponding lattice structures.

Theorem 2.6. An ideal of $\mathfrak{L}$ is a symmetric norm ideal if and only if it is a closed subspace of a maximal norm ideal. In particular maximal and minimal norm ideals are symmetric norm ideals.

Proof. Let $\left(\mathcal{G}^{\prime},\|\cdot\|_{g}\right)$ be a symmetric norm ideal, and let $\Phi$ be the symmetric norming function induced by $\|\cdot\|_{g}$. From Theorem $2.1, g \subseteq \delta_{\Phi}$. Since $\left(g,\|\cdot\|_{g}\right)$ is a symmetric norm ideal, for every $T \in \mathcal{G}$, we have $\|T\|_{g}=\lim _{P \in \varphi}\|P T\|_{\Phi}=\|T\|_{\Phi}$. Thus, from the fact that $\mathcal{I}$ is a Banach space, we conclude that $\mathcal{I}$ is a closed subspace of $\delta_{\text {. }}$. Conversely, suppose there exists $\Psi \in \boldsymbol{r}$ such that the ideal $\mathscr{g}$ of $\mathfrak{L}$ is a closed subspace of $\mathcal{S}_{\Psi}$. Then $\left(\mathcal{G},\|\cdot\|_{\Psi}\right)$ is clearly a norm ideal. Moreover, since $\lim _{P \in g}\|P T\|_{\Psi}=\|T\|_{\Psi}$, for every $T \in \mathcal{G}$, we conclude that $\left(\mathcal{G},\|\cdot\|_{\Psi}\right)$ is a symmetric norm ideal.

Remark 2.7. Let $p \geq 1, \Phi \in \pi$. We define $\Phi_{(p)}$ on $\mathfrak{F}$ by $\Phi_{(p)}(x)=\left[\Phi\left(x^{p}\right)\right]^{1 / p}$, where $x^{p}$ denotes the sequence whose terms are $x_{n}^{p}$. It can be easily checked that $\Phi_{(p)} \in \pi$, for every $p \geq 1$. Also, it readily follows that $\mathcal{G}_{\phi_{(p)}}=\left\{x \in \mathbb{R}: x^{p} \in \mathfrak{G}_{\phi}\right\}$, $\mathcal{G}_{(p)}^{(p)}=\left\{x \in \mathcal{R}: x^{p} \in \mathcal{G}_{\Phi}^{0}\right\}$.

Following $[11, \S 2]$ for each ideal $\mathscr{G}$ of $\mathcal{L}$, and each $p>0$ we shall denote by $g^{1 / p}$ the ideal defined by $\Lambda\left(g^{1 / p}\right)=[\Lambda(g)]^{1 / p}=\left\{x \in\left\{: x^{p} \in \Lambda(g)\right\}\right.$. Thus, $\delta_{(p)}=$ $\left(\delta_{\phi}\right)^{1 / p}, \delta_{\Phi(p)}^{0}=\left(\delta_{\Phi}^{0}\right)^{1 / p}, p \geq 1$.

Theorem 2.8. Let $\Phi \in \pi$ sucb that $\delta_{\Phi} \subseteq \mathcal{C}$. Then, for every $p>1, \delta_{\Phi} \subseteq$ $\left(\mathcal{S}_{\Phi}\right)^{1 / p}$ and $\mathcal{S}_{\Phi}^{0} \subsetneq\left(\mathcal{S}_{\Phi}^{0}\right)^{1 / p}$.

Proof. Let $p>1$. It is obvious that $\delta_{\phi} \subseteq\left(\delta_{\phi}\right)^{1 / p}$ and $\delta_{\Phi}^{0} \subseteq\left(\mathcal{S}_{\phi}^{0}\right)^{1 / p}$. On the other hand, since $\delta_{\Phi} \subseteq \mathcal{C}$, it follows that $\Phi \not \Phi_{\infty}$, and hence $\lim _{k \rightarrow \infty} \Phi\left[\sigma_{k}(u)\right]=\infty$ $\left(u_{n}=1, n=1,2, \ldots\right)$. Therefore

$$
\sup _{x \in \mathcal{J}}\left[\Phi(x) / \Phi_{(p)}(x)\right] \geq \sup _{k}\left(\Phi\left[\sigma_{k}(u) V / \Phi_{(p)}\left[\sigma_{k}(u)\right]\right)=\sup _{k}\left(\Phi\left[\sigma_{k}(u)\right]\right)^{1-1 / p}=\infty .\right.
$$

We conclude that $\left(\mathcal{S}_{\Phi}\right)^{1 / p} \supsetneq \mathcal{S}_{\phi},\left(\mathcal{S}_{\Phi}^{0}\right)^{1 / p} \supsetneq \mathcal{S}_{\phi}^{0}$ and our assertion follows. 
Theorem 2.9. Let $\mathcal{G}$ be an ideal of $\mathfrak{Q}$ such that $\mathscr{G} \subseteq \mathcal{C}$. If $\mathcal{G}=\mathcal{G}^{2}$, then. there does not exist $\Phi \in \boldsymbol{r}$ sucb that $\delta_{\Phi}^{0} \subseteq I \subseteq \mathcal{S}_{\Phi}$. In particular, $I$ cannot be a normed ideal.

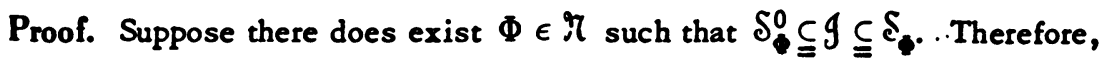
$\left(\delta_{\phi}^{0}\right)^{1 / 2} \subseteq g^{1 / 2} \subseteq\left(\delta_{\phi}\right)^{1 / 2}$. Since $\mathcal{G}=g^{2}$, it follows that $g=g^{1 / 2}$ and hence

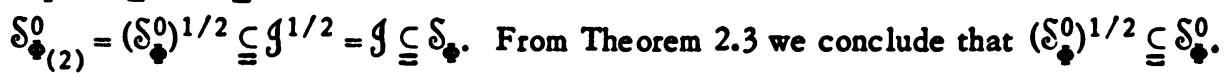
Since we always have $S_{\$}^{0} \subseteq\left(S_{\phi}^{0}\right)^{1 / 2}$ we deduce that $S_{\phi}^{0}=\left(S_{\phi}^{0}\right)^{1 / 2} \subseteq \varrho$ which contradicts Theorem 2.8 .

Remark 2.10. As a by-product of the above theorem we obtain the following conclusion: $U_{p \geq 1} C_{p}$ is not a norm ideal.

3. The K-conjugate ideal of an ideal. Given two ideals $\mathcal{I}$ and $\mathcal{I}$ of $\mathscr{L}$, the product $I \mathcal{I}$ of $I$ and $I$ is the linear span of the set $\{T S: T \in I, S \in I\}$. The following theorem can be proved with arguments similar to those used in the proof of [11, Theorem 2.1].

Theorem 3.1. Let $I$ and $I$ be two ideals of $\mathcal{L}$ Then the cbaracteristic set of the ideal 99 is related to the cbaracteristic sets of $\mathcal{I}$ and $I$ in the following natural way:

$$
\Lambda(g))=\Lambda(g) \Lambda(\mathcal{g})=\{x y: x \in \Lambda(g), y \in \Lambda(\mathcal{g})\} \text {. }
$$

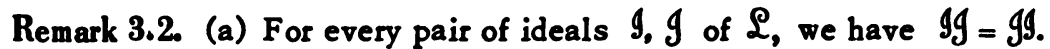

(b) If $\mathcal{I}$ is an ideal of $\mathcal{L}$ and $k$ is a positive integer then $\Lambda(g k)=[\Lambda(g)]^{k}=$ $\left\{x^{k}: x \in \Lambda(g)\right\}$ (cf. Remark 2.7).

Definition 3.3. Let $\mathcal{K}$ be an ideal of $\mathcal{L}$. For each ideal $\mathfrak{I}$ we define the K-conjugate of $\mathcal{G}$ as the ideal

$$
\mathfrak{g} \times\langle\mathcal{K}\rangle=\{T \in \mathcal{L}: S T \in \mathcal{K} \text {, for every } S \in \mathfrak{g}\} \text {. }
$$

Remark 3.4. We list below some elementary properties of the K-conjugate of an ideal $\mathrm{g}$.

(a) $g \times\langle K\rangle=\{T \in \mathcal{L}: T S \in \mathcal{K}$, for every $S \in 9\}$.

(b) $g(g \times\langle\mathcal{K}\rangle)=(g \times\langle\mathcal{K}\rangle) g \subseteq \mathcal{K}$ (as we shall see later this inclusion might be proper).

(c) $\Lambda(g \times\langle K\rangle)=\{x \in \mathbb{R}: x y \in \Lambda(\mathcal{K})$ for every $y \in \Lambda(g)\}$.

(d) $g \times\langle K\rangle$ is the largest of those ideals $g$ such that $g \mathscr{g} \subseteq \mathcal{K}$.

(e) $\Lambda(g \times\langle K\rangle)$ is the largest of those characteristic sets $\bar{\Xi}$ such that $\Lambda(g) \mathscr{J}$ $\subseteq \Lambda(\mathcal{K})$.

(f) $g \subseteq g \times<\langle K\rangle$.

(g) If $g \subseteq g$, then $g \times\langle K\rangle \subseteq g \times\langle K\rangle$.

(h) $g \times x \times\langle K\rangle=g \times\langle K\rangle$. 
(i) $\mathcal{K} \subseteq g \times\langle\mathcal{K}\rangle$, and $\mathfrak{Q} \times\langle\mathcal{K}\rangle=\mathcal{K}$.

(j) $A_{n}$ ideal $\mathcal{K}$ is called prime if for every ideal $g$ such that $g \nsubseteq \mathcal{K}$, we have $g \times\langle K\rangle=\mathcal{K}$; furthermore it follows that $\mathcal{K}$ is prime if and only if $g \times\langle\mathcal{K}\rangle=\mathcal{K}$ for every ideal $I$ containing $K$.

Theorem 3.5. Let $\mathcal{K}$ be an ideal of $\mathfrak{L}$ and let $\mathcal{G}=K^{1 / 2}$. Then $g \times\langle\mathcal{K}\rangle=\mathscr{G}$ and $\mathcal{G} g \times\langle K\rangle$. On the other band, if there exists a self $K$-conjugate ideal, then it must coincide with $\mathrm{g}$.

Proof. Since $g^{2}=\mathcal{K}$ it follows from Remark 3.4(d) that $g \subseteq g \times\langle K\rangle$. Then from Remark 3.4(g) $g \times\langle K\rangle \subseteq g \times\langle K\rangle$. Therefore, using Remark 3.4(b) and (f) we have $K=g g \subseteq g(g \times\langle K\rangle) \subseteq \bar{g}(g \times\langle K\rangle) \subseteq K$, and hence $g(g \times\langle K \mathcal{}(\mathcal{K})=K$. Since

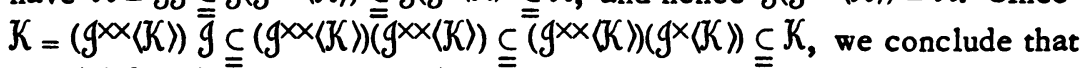
$(g \times<\langle K\rangle)^{2}=\mathcal{K}$ and hence $\mathscr{g} \times\langle\mathcal{K}\rangle=\mathscr{g}$, proving the first assertion. Now, if there exists $g$ such that $g=g \times\langle K\rangle$, it follows that $g \subseteq g$ because $g^{2}=g(g \times\langle K\rangle) \subseteq$

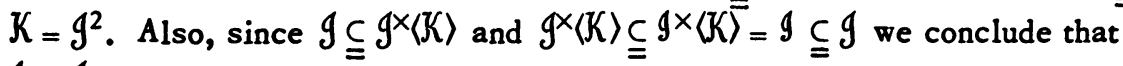
$\mathrm{I}=\mathrm{g}$. The proof of the theorem is completed.

In $\S 4$ we shall see that if $\Phi \in \Re$, then $\left(\delta_{\phi}\right)^{1 / 2}$ is the self $\oint_{\Phi}$-conjugate, and if $\Phi$ is not mono-normalizing, there does not exist any self $\delta_{\Phi}^{0}$-conjugate ideal.

Theorem 3.6. Let $\mathcal{I}$ and $\mathrm{I}$ be two ideals of $\mathcal{L}$. As usual, let $\mathrm{g} \vee \mathrm{g}$ denote the smallest ideal containing both $g$ and $g$. Then we bave $(g \vee g) \times\langle K\rangle=$ $g \times\langle K\rangle \cap g \times\langle K\rangle$. On the otber band $g \times\langle\mathcal{K}\rangle \vee g \times\langle\mathcal{K}\rangle \subseteq(9 \cap g) \times K$ :

Proof. From Remark 3.4(g) it is clear that $(g \vee g) \times\langle K\rangle \subseteq g \times\langle K\rangle \subseteq(g \cap g) \times\langle K\rangle$,

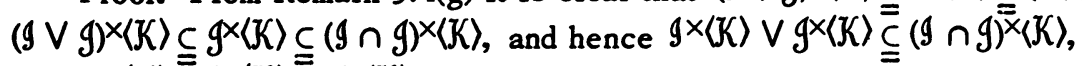
$(g \vee g) \times\langle K\rangle \cong g \times\langle K\rangle \cap g \times\langle K\rangle$. Thus, in order to complete the proof of the theorem it remains to prove that $g \times\langle\mathcal{\cap} \cap g \times\langle\mathcal{\subseteq} \cong(g \vee g) \times\langle K\rangle$. To this end let us observe that $\Lambda(\mathcal{G} \vee \mathscr{g})=\{x \vee y: x \in \Lambda(\mathcal{I}), y \in \Lambda(\mathcal{I})\}=\Lambda(g) \vee \Lambda(\mathcal{g})$, where $x \vee y$ denotes the sequence whose terms are $x_{n} \vee y_{n}, n=1,2, \ldots$ (cf. [1, Lemma 4.9]). Now it is immediate that if $\mathcal{X}$ is an ideal of $\mathscr{L}$, then $\Lambda(\mathscr{X})(\Lambda(\mathcal{I}) \vee \Lambda(g))=[\Lambda(\mathscr{X}) \Lambda(\mathscr{g})]$ $\vee[\Lambda(\mathscr{X}) \Lambda(g)]$, and hence $\mathscr{X}(g \vee g)=X g \vee \mathcal{X}$. Therefore

$$
\begin{aligned}
(g \vee g)\left(g \times\langle K\rangle \cap g^{\times}\langle\mathcal{K}\rangle\right) & =\left[g\left(g^{\times}\langle K\rangle \cap g^{\times}\langle K\rangle\right)\right] \vee\left[g\left(g^{\times}\langle K\rangle \cap g^{\times}\langle K\rangle\right)\right] \\
& \cong[g(g \times\langle K\rangle)] \vee[g(g \times\langle K\rangle)] \subseteq \mathcal{K} .
\end{aligned}
$$

From Remark 3.4(d) and the last chain of inclusions our assertion follows.

Theorem 3.7. If $\mathcal{C}\langle\mathcal{K}\rangle=\mathcal{K}$ and $g \nsubseteq \mathcal{K}$, then $g \times\langle\mathcal{K}\rangle \subsetneq \mathcal{C}$.

Proof. Since $\mathscr{I} \varsubsetneqq \mathcal{K}$, we see that $\mathcal{K} \neq \mathscr{L}$, and hence $\mathcal{C}\langle K\rangle=\mathcal{K}$ implies that $\mathcal{K} \cong \mathcal{C}$. Now let $T \in \mathcal{I}$ such that $T \notin \mathcal{K}$. Since $\mathcal{C}\langle\mathcal{K}\rangle=\mathcal{K}$, there exists $S \in \mathcal{C}$ such that $T S \notin \mathcal{K}$. It follows that $T \notin g \times\langle\mathcal{K}\rangle$ which implies that $g \times\langle\mathcal{K}\rangle \sqsubseteq \mathcal{C}$. 
Theorem 3.8. Let $\mathcal{K}$ be a maximal norm ideal. Then $\mathcal{C} \times\langle\mathcal{K}\rangle=\mathcal{K}$.

Proof. Let $\Phi \in \boldsymbol{r}$ such that $\mathcal{K}=\delta_{\phi}$. If $\delta_{\Phi}=\mathscr{L}$ there is nothing to prove. Therefore, we may assume that $\delta_{\Phi} \subseteq \mathcal{C}$. From Remark 3.4(c) it suffices to prove that for every $x \in \mathbb{R}$ such that $x \notin \mathcal{E}_{\phi}$ there exists $y \in \Lambda(\mathcal{C})$ such that $x y \notin \mathcal{E}_{\varnothing}$. Thus, suppose $x \in \mathfrak{R}$ and $x \notin \mathcal{G}_{\Phi^{\circ}}$. Then there exists $k_{1}$ such that $\Phi\left[\sigma_{k_{1}}(x)\right] \geq 1$. Since the tail $r_{k_{1}}(x)\left(=\left\{x_{1+k_{1}}, x_{2+k_{1}}, \cdots\right\}\right)$ of $x$ is not in $\delta_{\phi}$, there exists $k_{2}$ such that $\Phi\left[\sigma_{k_{2}}\left(r_{k_{1}}(x)\right)\right] \geq 2^{2}$. Continuing in this manner, let $k_{n+1}$ be chosen so that $\Phi\left[\sigma_{k_{n}+1}\left(r_{k_{n}}(x)\right)\right] \geq(n+1)^{2}$. Now define $y \in \mathfrak{R}$ as follows: $y_{n}=1,1 \leq n \leq k_{1}$, $y_{n}=1 / 2, k_{1}+1 \leq n \leq k_{1}+k_{2}, y_{n}=1 / 3, k_{1}+k_{2}+1 \leq n \leq k_{1}+k_{2}+k_{3}$, etc. It is clear that $y \in \Lambda(\mathcal{C})$ and that

$$
\lim _{k \rightarrow \infty} \Phi\left[\sigma_{k}(x y)\right] \geq \lim _{n \rightarrow \infty} \Phi\left(\sigma_{k_{n+1}}\left[\tau_{k_{n}}\left(\frac{x}{n+1}\right)\right]\right)=\infty .
$$

We conclude that $x y \notin G_{\phi}$, as desired.

Remark 3.9. (a) Let $\Phi \in \boldsymbol{r}$ be a non mono-normalizing function. From Theorem 3.8 we have $\mathcal{C}\left\langle\left\langle\mathcal{S}_{\phi}\right\rangle=\delta_{\phi}\right.$. On the other hand, if $x \in \mathcal{E}_{\phi}$ and $y \in \Lambda(\mathcal{C})$ we see that for each $m=1,2, \ldots$ we have $\Phi\left[r_{m}(x y)\right] \leq y_{m} \Phi\left[r_{m}(x)\right] \leq y_{m} \Phi(x)$, and hence $\lim _{m \rightarrow \infty} \Phi\left[r_{m}(x y)\right]=0$. Therefore $\mathcal{C}\left(\mathcal{C}^{x}\left\langle\delta_{\phi}\right\rangle \subseteq \delta_{\Phi}^{0} \varsubsetneqq \delta_{\Phi}^{m}\right.$. Thus, this is an example in which the inclusion in Remark 3.4(b) is proper.

(b) The inclusion in the second statement of Theorem 3.6 might be proper. Let $g$ and $g$ be two ideals of $\mathcal{L}$ such that $g \cap g=\mathcal{C}_{1}, \mathcal{C}_{1} \varsubsetneqq g$ and $\mathcal{C}_{1} \varsubsetneqq g$ (cf. [10, Remark 4.5]). From Theorem $3.7 g \times\left\langle\mathcal{C}_{1}\right\rangle \varsubsetneqq \mathcal{C}, g \times\left\langle\mathcal{C}_{1}\right\rangle \varsubsetneqq \mathcal{C}$, and hence $g \times\left\langle\mathcal{C}_{1}\right\rangle \vee g \times\left\langle\mathcal{C}_{1}\right\rangle \subseteq \mathcal{C}$. However $(g \cap g) \times\left\langle\mathcal{C}_{1}\right\rangle=\mathfrak{L}$.

4. The K-conjugate of norm ideals. In this section we shall be primarily concerned with the $\mathcal{K}$-conjugate of maximal and minimal norm ideals, where $\mathcal{K}$ is either a maximal or a minimal norm ideal.

Definition 4.1. Let $\Phi, \Psi \in \Re$. We define the function $\Psi_{\Phi}^{*}$ on $\mathfrak{F}$ by the formula

$$
\Psi_{\phi}^{*}(x)=\sup _{y \in \mathfrak{J}_{;} ; y_{1} \neq 0}[\Phi(x y) / \Psi(y)] \text {. }
$$

Remark 4.2. It follows that $\Psi_{\phi}^{*} \in \pi$, for every $\Phi, \Psi \in \Pi_{\text {. The only property }}$ of symmetric norming functions that $\Psi_{\Phi}^{*}$ does not obviously satisfy is the fifth one. This is a consequence of the following fact [8, Lemma 3]. If $z \in \mathbb{R}$ and $x, y \in \mathfrak{F}$ such that $\sum_{n=1}^{m} x_{n} \leq \Sigma_{n=1}^{m} y_{n^{\prime}} m=1,2, \cdots$, then $\sum_{n=1}^{m} x_{n} z_{n} \leq \sum_{n=1}^{m} y_{n^{\prime}} z_{n^{\prime}} m=$ $1,2, \ldots$. The function $\Psi_{\Phi}^{*}$ will be called the conjugate function of $\Psi_{\text {relative to }}$ $\Phi$, or simply the $\Phi$-conjugate of $\Psi$. The following different characterizations of the $\Phi$-conjugate of $\Psi$ are easy to verify:

$$
\Psi_{\Phi}^{*}(x)=\sup _{y \in \mathbb{B} ; \Downarrow(y) \leq 1} \Phi(x y)=\sup _{y \in \mathcal{S}_{\Psi}^{0} ; \Psi(y) \leq 1} \Phi(x y)=\sup _{y \in \mathcal{S}_{\Psi} ; \psi(y) \leq 1} \Phi(x y) .
$$


The following theorem is the central result of the present section.

Theorem 4.3. Let $\Phi, \Psi$ be in $\pi$. Then the following statements are valid:

(a) $j_{\Psi} x\left\langle S_{\Phi}\right\rangle=\delta_{\Psi}^{0} \times\left\langle S_{\Phi}\right\rangle=S_{\Psi} \times\left\langle S_{\Phi}^{0}\right\rangle=\delta_{\Psi}^{*}$.

(b) Let $\stackrel{\Upsilon}{\chi}$ be one of the sets: (I) $\left\{X \in \mathcal{F}:\|X\|_{\Psi} \leq 1\right\}$, (II) $\left\{X \in \mathcal{S}_{\Psi}^{0}:\|X\|_{\Psi} \leq 1\right\}$, (III) $\left\{X \in S_{\Psi}:\|X\|_{\Psi} \leq 1\right\}$.

Then, for every $T \in \mathcal{S}_{\Psi_{\Phi}^{*} \text { we bave }}$

$$
\|T\|_{\Psi^{*}}=\sup _{X \in \mathscr{X}}\|T X\|_{\Phi}=\sup _{X \in \mathscr{X}}\|X T\|_{\Phi}=\sup _{X \in \mathscr{X}} \Phi\left[s(T)_{s}(X)\right] \text {. }
$$

(c) (Generalized Holder inequality). For every $S \in \mathcal{S}_{\boldsymbol{\Psi}}, T \in \mathcal{S}_{\boldsymbol{\Psi}^{*}}$ we bave $\max \left(\|T S\|_{\Phi^{\prime}},\|S T\|_{\Phi}\right) \leq \Phi\left[s(S)_{s}(T)\right] \leq\|S\|_{\boldsymbol{\psi}}\|T\|_{\boldsymbol{\Psi}_{\boldsymbol{\Phi}^{*}}}$

(d) If $\mathcal{S}_{\Phi} \subseteq \mathcal{C}$ and $\mathcal{S}_{\Psi} \varsubsetneqq \mathcal{S}_{\Phi}$ (and bence $\left.\mathcal{S}_{\Psi}^{0} \not \mathcal{S}_{\phi}^{0}\right)$, then $\mathcal{S}_{\Phi} \subseteq \mathcal{S}_{\Psi_{\phi}^{*}} \subseteq \mathcal{C}$. we have

Proof. We first establish (c). Let $S \in \mathcal{S}_{\Psi}, T \in \mathcal{S}_{\psi_{\Phi}^{* \prime}}$ then for every $k=1,2, \ldots$

$$
\begin{aligned}
\Phi\left(\sigma_{k}\left[s(S)_{s}(T)\right]\right) & =\Phi\left(\sigma_{k}[s(S)] \sigma_{k}[s(T)]\right) \leq \Psi\left(\sigma_{k}[s(S)]\right) \Psi_{\phi}^{*}\left(\sigma_{k}[s(T)]\right) \\
& \leq \Psi[s(S)] \Psi_{\Phi}^{*}[s(T)]=\|S\|_{\Psi}\|T\|_{\Psi_{\Phi}^{*}}
\end{aligned}
$$

Therefore $\Phi\left[s(S)_{s}(T)\right] \leq\|S\|_{\Psi}\|T\|_{\Psi_{\Phi}^{* *}}$ Since the first inequality in part (a) follows from Horn's inequality, the proof of part (c) is completed. We also deduce that $\delta_{\psi_{\Phi}^{*}} \subseteq \int_{\Psi}^{X}\left\langle\mathcal{S}_{\Phi}^{0}\right\rangle$. The proof of (a) will be finished after showing that $\mathcal{S}_{\Psi}^{0 \times}\left\langle\mathcal{S}_{\Phi}\right\rangle \subseteq \mathcal{S}_{\boldsymbol{\psi}_{\Phi}^{*}}$ To this end let $T \in S_{\Psi}^{0 \times}\left\langle\delta_{\Phi}\right\rangle$ and let $\left\{P_{n}\right\}$ be an increasing sequence in $\mathcal{P}$ tending strongly to the identity operator such that rank $P_{n}=n, n=1,2, \ldots$ Next, we define for $k=1,2, \ldots$ the linear transformation $\psi_{k}: \delta_{\Psi}^{0} \rightarrow \delta_{\Phi}$ by the identity $\psi_{k}(S)=P_{k} T S, S \in \mathcal{S}_{\Psi}^{0}$. Then $\left\|\psi_{k}(S)\right\|_{\Phi}=\Phi\left[s\left(P_{k} T S\right)\right] \leq \Phi\left(\sigma_{k}[s(T S)]\right) \leq \Phi[s(T S)]=$ $\|T S\|_{\Phi}$ and $\left\|\psi_{k}(S)\right\|_{\Phi} \leq\|S\|_{\Psi}\left\|P_{k} T\right\|_{\psi^{*}}, k=1,2, \ldots$ Therefore, from the uniform boundness principle it follows that

$$
\sup _{s \in \delta_{\Psi}^{0} ;\|s\|_{\Psi} \leq 1}\left\|P_{k} T S\right\|_{\Phi} \leq \gamma,
$$

for some $\gamma>0, k=1,2, \ldots$, and hence

$$
\sup _{s \in \mathcal{S}_{i}\|s\|_{\Psi} \leq 1}\|T S\|_{\Phi} \leq \gamma
$$

Now let $X \in \mathcal{F}$, and let $T=V Q$ (where $Q=\sqrt{T{ }^{*} T}$ and $V$ is a partial isometry in $\mathscr{L}$ ) be the polar decomposition of the operator $T$. It is easy to see that there exists a positive operator $R \in \mathcal{F}$ such that $R Q=Q R, s(R)=s(X)$ and $s(R Q)=$ $s(R) s(Q)$. Now let $W$ be an isometry in $\mathscr{L}$ such that $W V^{*} V=V$ whenever $T$ (and hence $Q$ ) is in $\mathcal{F}$, and let $W=V$ otherwise. Then $R Q=R W^{*} W Q=R W^{*} W V^{*} V Q=$ 
$R W^{*} V Q=R W^{*} T$. Now let $Y=R W^{*}$. It follows that $\Phi[s(X T)] \leq \Phi\left[s(X)_{s}(T)\right]=$ $\Phi[s(R) s(Q)]=\Phi[s(R Q)]=\Phi[s(Y T)]$. Since $\Psi[s(X)]=\Psi[s(Y W)] \leq \Psi[s(Y)]$ we conclude that

$$
\sup _{s \in \mathcal{F}_{;}\|s\|_{\Psi} \leq 1}\|S T\|_{\Phi}=\sup _{s \in \mathcal{F}_{i}\|s\|_{\boldsymbol{\Psi}} \leq 1} \Phi\left[s(S)_{s}(T)\right]
$$

In a similar way it can be proved that

$$
\sup _{s \in \mathcal{F}_{;}\|s\|_{\Psi} \leq 1}\|T S\|_{\Phi}=\sup _{s \in \mathcal{I}_{;}\|s\|_{\Psi} \leq 1} \Phi\left[s(S)_{s}(T)\right] .
$$

From the formulas just proved we conclude that

$$
\|T\|_{\Psi_{\Phi}^{*}}=\sup _{s \in \mathcal{F}_{i}\|s\|_{\Psi} \leq 1} \Phi\left[s(S)_{s}(T)\right] \leq \gamma,
$$

and hence $T \in S_{\mathcal{H}^{* \bullet}}$ Therefore $\mathcal{S}_{\Psi}^{0 \times}\left\langle\mathcal{S}_{\phi}\right\rangle \subseteq \mathcal{S}_{\psi_{0}^{*}}$, as desired. Also, employing Remark 4.2, the proof of (b)(I) follows. Since (b)(II) and (b)(III) are consequences of (b)(I), (c) and Remark 4.2, the proof of (b) is completed. Finally part (d) is a direct consequence of Theorem 3.7.

Corollary 4.4. Let $\Phi, \Psi \in \Re$ and let $g$ and $\mathcal{K}$ be two ideals of $\mathcal{L}$ sucb that

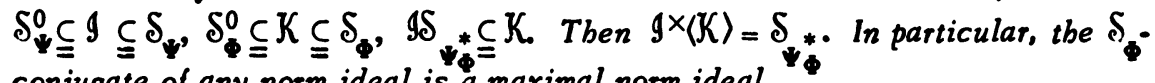
conjugate of any norm ideal is a maximal norm ideal.

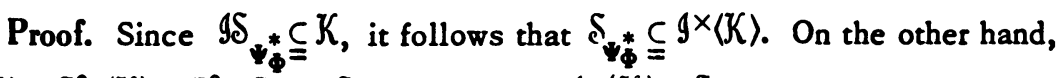
$g^{*}(K\rangle \subseteq \mathcal{S}_{\Psi}^{0 \times}\langle K\rangle \subseteq S_{\Psi}^{0 \times}\left\langle\mathcal{S}_{\Phi}\right\rangle=S_{\Psi_{\Phi}^{* \cdot}}$ Therefore $g \times\langle\mathcal{K}\rangle=S_{\Psi_{\phi}^{*}}$.

Theorem 4.5. Let $\Phi \in \boldsymbol{r}$, and let $p>1, q>1$ such that $1 / p+1 / q=1$. Then $\left(S_{\Phi}\right)^{1 / p}$ and $\left(S_{\Phi}\right)^{1 / q}$ are $S_{\Phi}$-conjugate one of each otber. Also $\left[\left(S_{\Phi}^{0}\right)^{1 / p}\right]^{\times}\left\langle\delta_{\Phi}^{0}\right\rangle=$ $\left(\mathcal{f}_{\Phi}\right)^{1 / q}$. If $\Phi_{(p)}$ and $\Phi_{(q)}$ are as in Remark 2.7, then $\left(\Phi_{(q)}\right)_{\Phi}^{*}=\Phi_{(p)}$.

Proof. From Theorem 4.3 it clearly suffices to prove the last statement. Let $x, y \in \mathfrak{F} x_{1} \neq 0, y_{1} \neq 0$ and let $x^{\prime}, y^{\prime} \in \mathfrak{F}$ such that $x^{\prime}=x / \Phi_{(p)}(x), y^{\prime}=y / \Phi_{(q)}(y)$.

From a well-known inequality it follows that $x_{n}^{\prime} y_{n}^{\prime} \leq\left(x_{n}^{\prime}\right)^{p} / p+\left(y_{n}^{\prime}\right)^{q} / q, n=1,2, \ldots$, and therefore $\Phi\left(x^{\prime} y^{\prime}\right) \leq \Phi\left[\left(x^{\prime}\right)^{p}\right] / p+\Phi\left[\left(y^{\prime}\right)^{q}\right] / q \leq 1 / p+1 / q=1$. We conclude that $\Phi(x y) \leq \Phi_{(p)}(x) \Phi_{(q)}(y)$ and hence $\left(\Phi_{(q)}\right)_{\Phi}^{*}(x) \leq \Phi_{(p)}(x)$, for every $x \in \mho_{\text {. In order }}$ to prove the reverse inequality, we choose, for a given $x \in \mathfrak{F}, x_{1} \neq 0$ a sequence $y \in \mathfrak{F}$ such that $y=x^{p-1} /\left[\Phi_{(p)}(x)\right]^{p / q}$. It follows that $\Phi_{(q)}(y)=$ $\left[\Phi\left(x^{q(p-1)}\right)\right]^{1 / q} /\left[\Phi\left(x^{p}\right)\right]^{1 / q}=1$. On the other hand $\Phi(x y)=\Phi\left(x^{p}\right) /\left[\Phi\left(x^{p}\right)\right]^{1 / q}=$

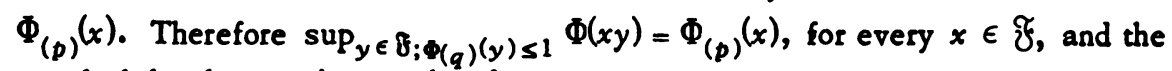
proof of the theorem is completed.

Corollary 4.6. Let $\Phi \in \boldsymbol{r}$. Then $\left(\oiint_{\Phi}\right)^{1 / 2}$ is the self $\S_{\Phi}$-conjugate ideal. Moreover, if $\Phi$ is not mono-normalizing, then there does not exist any self $\boldsymbol{S}_{\Phi}^{0}$ conjugate ideal. 
Remark 4.7. (a) Let $\Phi \in \boldsymbol{H}$ be a non mono-normalizing function. From Theorem 4.5 we see that $\left[\left(\delta_{\phi}^{0}\right)^{1 / 2}\right]^{\times}\left\langle\delta_{\phi}\right\rangle=\left(\delta_{\phi}\right)^{1 / 2}=\left[\left(\delta_{\phi}^{0}\right)^{1 / 2}\right]^{\times}\left\langle\left(S_{\phi}^{0}\right\rangle\right.$. Therefore, $\left(\delta_{\Phi}^{0}\right)^{1 / 2}\left[\left(\delta_{\Phi}^{0}\right)^{1 / 2}\right]^{\times}\left\langle\delta_{\phi}\right\rangle \subseteq \delta_{\phi}$ and we have found another example in which the inclusion in Remark 3.4(b) is proper.

(b) Let $\Phi, \Psi \in \mathcal{H}$. Then a necessary and sufficient condition for the validity of $S_{\Psi}^{x \times}\left\langle S_{\Phi}\right\rangle=S_{\Psi}$ is that $\Psi$ be equivalent to $\Omega_{\phi}^{*}$, for some $\Omega \in \Re$.

(c) From the results of [12, Chapter 5] and [5, Chapter 3] it follows that, if $S_{\Phi}=\mathcal{C}_{1}$, then $S_{\Phi^{*}}$ is the topological dual of $\mathcal{S}_{\Psi}^{0}$ and the Köthe-dual of $\mathcal{S}_{\Psi^{\prime}}$, and $\Psi_{\Phi}^{* *}=\Psi$. Moreover, from Theorem 4.3 we conclude that if $\delta_{\phi}=\mathcal{C}_{1}$, then $\delta_{\Psi^{*}}$ is the Köthe-dual of $\mathcal{S}_{\Psi}^{0}$ and can be embedded (topologically and isomorphically) into the topological dual of $\delta_{\psi}$. This embedding can be obtained via the nondegenerate bilinear form trace $(S T), S \in \mathcal{S}_{\Psi^{\prime}} T \in \mathcal{S}_{\boldsymbol{\Psi}_{\Phi}^{*}}$.

(d) The set of symmetric norm ideals of $\mathscr{\&}$ has a natural lattice structure. Let $\left(g,\|\cdot\|_{g}\right)$ and $\left(g,\|\cdot\|_{g}\right)$ be two symmetric norm ideals. We define for each $T \in \mathcal{G} \cap \mathcal{G},\|T\|_{g \cap g}=\max \left(\|T\|_{g},\|T\|_{g}\right)$. It is easy to check that $\left(g \cap g,\|\cdot\|_{g} \cap\right)$ is a symmetric norm ideal and we write $\left(g \cap g,\|\cdot\|_{g \cap g}\right)=\left(g,\|\cdot\|_{g}\right) \wedge\left(g,\|\cdot\|_{g}\right)$. On the other hand, since $(g \vee g) \times\left\langle\mathcal{C}_{1}\right\rangle=g \times\left\langle\mathcal{C}_{1}\right\rangle \cap g \times\left\langle\mathcal{C}_{1}\right\rangle$ we see that $(g \vee g) \times\left\langle\mathcal{C}_{1}\right\rangle$ is a symmetric norm ideal, and hence $(g \vee g) \times\left\langle C_{1}\right\rangle$ is the smallest maximal norm ideal containing $g \cup g$. Let $\Omega \in \pi$ such that $\delta_{\Omega}=(g \vee g)^{\times \times}\left\langle\mathcal{C}_{1}\right\rangle$. We define $\left(g,\|\cdot\|_{g}\right) \vee\left(g,\|\cdot\|_{g}\right)$ to be the closure in $\delta_{\Omega}$ of $g \vee g$, and we provide this norm ideal with the norm $\|\cdot\|_{\Omega}$. Therefore, by definition $\left(g,\|\cdot\|_{g}\right) \vee\left(g,\|\cdot\|_{g}\right)$ is the smallest symmetric norm ideal containing $g \cup g$ and $\left(g,\|\cdot\|_{g}\right) \wedge\left(\mathscr{g},\|\cdot\|_{g}\right)$ is the largest symmetric norm ideal contained in $I \cap \mathrm{g}$. In particular, the set of maximal norm ideals and the set of minimal norm ideals constitute lattices under inclusion with the lattice operations described above. It follows that none of these lattices is linearly ordered (Corollaries 5.5, 5.6 and [10, Theorem 3.7]). We also see that the mappings $\widetilde{\Phi} \rightarrow \delta_{\Phi}$ and $\widetilde{\Phi} \rightarrow \delta_{\Phi}^{0}$ are lattice preserving. B. S. Mityagin änd A. S. Shvartz $[9, \$ 7]$ proved that there exists a bijective correspondence between the symmetric norm ideals of $\mathfrak{L}$ and the symmetric norm ideals of $l^{\infty}$ (this correspondence turns out to be the restriction to the class of symmetric norm ideals of the Calkin lattice isomorphism). The above observation implies that the Mityagin and Shvartz correspondence is a lattice isomorphism.

(e) Let $0 \neq T \in \mathscr{L}$ and denote by $(T)$ the principal ideal generated by $T$. Then for every $\Phi \in \boldsymbol{r},(T)^{\times}\left\langle S_{\Phi}\right\rangle$ is a maximal norm ideal. If $\pi_{n}=s_{n}(T) / s_{1}(T), n=$ $1,2, \cdots$, and $\Phi_{\pi}$ is defined on $\mathfrak{F}$ by $\Phi_{\pi}(x)=\Phi(\pi x)$, then $\Phi_{\pi} \in \Re$ and $(T)^{\times}\left\langle\delta_{\Phi}\right\rangle=S_{\Phi_{\pi}}$. The case $\delta_{\Phi}=\mathcal{C}_{1}$ is especially interesting. In fact, $(T)^{\times}\left\langle\mathcal{C}_{1}\right\rangle$ is the maximal (and minimal) norm ideal $\delta_{\pi}$ introduced in [6]. A symmetric norming function for the maximal ideal $\left.(T)^{\times}<C_{1}\right\rangle$ is given by $\sup _{k}\left(\sum_{n=1}^{k} x_{n} / \sum_{n=1}^{k} \pi_{n}\right)$, $x \in \mathfrak{F}$. If $T \in \mathcal{C}$, and $T \notin \mathcal{C}_{1}$ this symmetric norming function is not mono-normalizing. 
Therefore the corresponding maximal norm ideal $\boldsymbol{S}_{\mathbf{n}}$ is nonseparable. If follows that the minimal norm ideal $\mathcal{S}_{\mathbf{n}}^{0}$ corresponding to $\mathcal{S}_{\mathbf{n}}$ can be characterized by

$$
\delta_{\Pi}^{0}=\left\{s \in \mathcal{S}_{\mathbb{n}}: \limsup _{k \rightarrow \infty} \frac{\sum_{n=1}^{k} s_{n}(s)}{\sum_{n=1}^{k} \pi_{n}}=0\right\} \text {. }
$$

The ideals of $\mathcal{S}_{\boldsymbol{n}}$ and $\boldsymbol{S}_{\boldsymbol{\Pi}}^{0}$ were already introduced in [6] and the above mentioned facts about the ideals $\mathcal{S}_{\pi}, \mathcal{S}_{\mathbf{n}}$ and $\mathcal{S}_{\mathbf{n}}^{0}$ are proved in detail in [5, Chapter 3]. These properties will be used in the following theorem.

Theorem 4.8. Minimal norm ideals are not principal ideals.

Proof. Let $\mathcal{G}$ be a minimal norm ideal and suppose there exists $T \in \mathcal{L}$ such that $\mathcal{J}$ coincides with the principal ideal $(T)$ generated by $T$. We first assume that $\mathcal{C}_{1} \subseteq g$. Then $T \notin \mathcal{C}_{1}$ and from Remark 4.7(e) it follows that

$$
\begin{aligned}
(T)^{\infty \times}\left\langle\mathcal{C}_{1}\right\rangle=\delta_{\mathbf{n}}=\left\{S \in \mathcal{L}: \underset{k \rightarrow \infty}{\lim \sup } \frac{\sum_{n=1}^{k} s_{n}(S)}{\sum_{n=1}^{k} \pi_{n}}<\infty,\right. \\
\text { where } \left.\pi_{n}=\frac{s_{n}(T)}{s_{1}(T)}, n=1,2, \ldots\right\} .
\end{aligned}
$$

Since $(T)$ is assumed to be a norm ideal there must exist $\Phi \in \boldsymbol{K}$ such that $(T)=\mathcal{S}_{\Phi}^{0}$. From the fact that $\mathcal{S}_{\Phi}^{0 \times \times}\left\langle\mathcal{C}_{1}\right\rangle=\mathcal{S}_{\Phi}$ (Remark $\left.4.7(c)\right)$ we deduce that

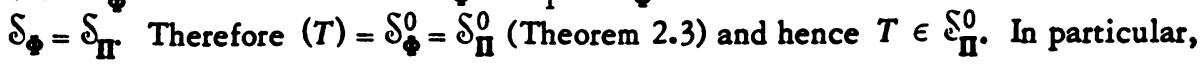
we conclude that

$$
0=\underset{k \rightarrow \infty}{\lim \sup _{n}} \sum_{n=1}^{k} s_{n}(T) / \sum_{n=1}^{k} \pi_{n}=s_{1}(T)=\|T\|,
$$

which is a contradiction. On the other hand, if $\mathcal{J}=\mathcal{C}_{1}$ and $T \in \mathcal{J}$, then from $[10$, Theorem 4.6] there exists an ideal $\mathcal{K} \subsetneq \mathcal{J}$ such that $T \in \mathcal{K}$ and hence $\mathcal{J} \neq(T)$. The proof of the theorem is complete.

5. The ideals $\mathcal{S}(f ; \mathcal{J})$ and $\mathscr{L}(f ; \mathcal{G})$. Let $\mathcal{J}$ be an ideal of $\mathcal{L}$ and, for $p>0$, let $f_{(p)}$ be the function defined on the nonnegative real line by $f_{(p)}(\xi)=\xi^{p}, \xi \geq 0$. Then the pth root of $\mathcal{J}$ (Remark 2.9) can also be defined as $\Lambda\left(\mathcal{f}^{1 / p}\right)=\left\{x \in \mathbb{R}: f_{(p)} \circ\right.$ $x \in \Lambda(\mathscr{g})\}$, where $f_{(p)} \circ x$ denotes the composition of the function $f_{(p)}$ and the sequence $x$ (i.e., $\left.\left(f_{(p)} \circ x\right)_{n}=x_{n^{\prime}}^{p} n=1,2, \ldots\right)$. There are many other functions that can be used together with an ideal $g$ to produce new ideals. Following [1] we adopt the following terminology.

Definition 5.1. We shall say that a nondecreasing nonnegative function $f$ defined on the nonnegative real line is an admissible function whenever $f(\xi)>0$, 
for every $\xi>0$ and $f(0)=0=\lim _{\xi \rightarrow 0} f(\xi)$. The class of all admissible functions will be denoted by $\mathfrak{A}$.

Remark 5.2. Given an ideal $\mathcal{J}$ of $\mathcal{L}$ and $f \in \mathbb{Q}$, the set $\{x \in \mathbb{R}: f \circ x \in \Lambda(\mathcal{J})\}$ is not in general a characteristic set. However, it follows that [10] the smallest characteristic set containing $\{x \in \mathbb{R}: f \circ x \in \Lambda(\mathscr{J})\}$ is given by

$$
G(f ; \Lambda(g))=\{x \in \mathbb{R}: f \circ(a x) \in \Lambda(g), \text { for some } a>0\}
$$

and the largest characteristic set contained in $\{x \in \mathbb{R}: f \circ x \in \Lambda(\mathcal{J})\}$ is defined by

$$
\mathscr{D}(f ; \Lambda(\mathcal{g}))=\{x \in \mathfrak{R}: f \circ(a x) \in \Lambda(\mathcal{g}) \text {, for all } a>0\} \text {. }
$$

It is observed in [10] that if $f \in \mathbb{Q}$ is a mono-generating function, that is if there exist $a>1, \gamma>0, \epsilon>0$ such that $f(a \xi) \leq \gamma f(\xi), 0 \leq \xi \leq \epsilon$, then $\mathcal{S}(f ; \Lambda(\mathcal{J}))=\mathscr{I}(f ; \Lambda(\mathcal{J}))$, and hence $\{x \in\{: f \circ x \in \Lambda(g)\}$ is a characteristic set. Moreover, the above condition is necessary whenever $\mathcal{J}=\mathcal{C}_{1}$. We let $\mathcal{S}(f ; \mathcal{J})$ and $\mathscr{I}(f ; \mathcal{J})$ be the ideals of $\mathcal{L}$ defined by

$$
\Lambda[S(f ; g)]=G(f ; \Lambda(g)), \quad \Lambda[D(f ; g)]=D(f ; \Lambda(g)) .
$$

It follows that

$$
\begin{aligned}
& \mathcal{S}(f ; \mathscr{g})=\left\{T \in \mathscr{L} ; f\left(a \sqrt{T^{*} T}\right) \in \mathscr{I}, \text { for some } a>0\right\}, \\
& \mathcal{D}(f ; \mathscr{I})=\left\{T \in \mathscr{L}: f\left(a \sqrt{T^{*} T}\right) \in \mathcal{I}, \text { for every } a>0\right\} .
\end{aligned}
$$

As in [10] we define the following order relation and equivalence relation on $\mathcal{Q}$. Let $f_{g} \in \mathbb{Q}$, then $f<g$ if and only if there exist positive numbers $\alpha, \beta, \gamma$ and $\epsilon$ such that $f(\alpha \xi) \leq \gamma_{g}(\beta \xi), 0 \leq \xi \leq \epsilon ; f \sim g$ if and only if $f<g$ and $g<f$. It is easy to prove that if $f<g$ and $\mathcal{G}$ is an ideal of $\mathcal{L}$, then $S(g ; \mathcal{I}) \subseteq S(f ; \mathcal{J})$ and $\mathscr{L}(g ; \mathcal{I}) \subseteq \mathscr{I}(f ; \mathcal{J})$. Therefore, if $f \sim g$, then $\mathcal{S}(f ; \mathcal{I})=\mathcal{S}(g ; \mathcal{I})$ and $\mathscr{I}(f ; \mathscr{I})=\mathscr{L}(g ; \mathcal{I})$. All these conditions become necessary and sufficient when $\mathscr{I}=C_{1}[10$, Theorem 2.12].

In the present section we study the ideals $S(f ; \mathscr{I})$ and $\mathscr{I}(f ; \mathcal{I})$ in the case that $\mathcal{G}$ is either a maximal or a minimal norm ideal and $f$ is a convex admissible function. If $g \in \mathbb{Q}$ and $g(\xi) / \xi, \xi>0$ defines a function in $\mathcal{Q}$, then it is easy to see that $[10, \text { Corollary } 3.5]_{g}$ is equivalent to a convex admissible function $f$ satisfying:

(*) $f(1)=1$,

(**) $\lim _{\xi \rightarrow 0} f(\xi) / \xi=0$,

$(* * *) \lim _{\xi \rightarrow \infty} f(\xi) / \xi=\infty$.

From the above remark we have $S(f ; \mathcal{I})=S(g ; \mathcal{I})$ and $\mathscr{D}(f ; \mathcal{I})=\mathscr{L}(g ; \mathcal{I})$, for every ideal $\mathscr{S}$ of $\mathcal{L}$. On the other hand, if 8 is a convex function in $\mathcal{Q}$ such that $g(\xi) / \xi, \xi>0$ does not define an admissible function, then $g \sim f_{(1)}$ and hence $\mathcal{S}(g ; \mathcal{I})=\mathcal{J}=\mathscr{L}(g ; \mathfrak{J})$, for every ideal $\mathcal{I}$ of $\mathcal{L}$. Since this case is irrelevant, and 
the normalizations $(*)$ and $(* * *)$ of a convex admissible function $f$ do not affect the ideals they generate, throughout $\$ 5$ and $\$ 6$ we shall restrict our considerations to convex admissible functions $f$ satisfying $(*),(* *)$ and $(* * *)$, because the arguments are simpler and more relevant for them. The set of these admissible functions will be denored by $\mathfrak{Q}_{\boldsymbol{c}}$.

Lemma 5.3. Let $\Phi \in \boldsymbol{r}$ and $f \in \mathbb{C}_{c}$. Then the function $\Phi$, defined on $\mathfrak{F}$ by $\Phi_{f}(x)=\inf _{\Phi[f \circ(x / \rho)] \leq 1} \rho$ is in $r_{\text {. . Moreover }} \Phi\left[f \circ\left(x / \Phi_{f}(x)\right)\right]=1$ for every $x \in \mathfrak{F}$.

Proof. The last statement follows immediately from the continuity properties of the function $\Phi$. On the other hand, it readily follows that $\Phi$, satisfies properties (i), (iii) and (iv) of symmetric norming functions. In order to prove (ii), let $x, y \in \mathfrak{F}$ such that $a=\Phi_{f}(x) \neq 0, \beta=\Phi_{f}(y) \neq 0$. Then,

$$
\begin{aligned}
\Phi\left[f \circ\left(\frac{x+y}{\alpha+\beta}\right)\right] & =\Phi\left[f \circ\left(\frac{\alpha}{\alpha+\beta} \frac{x}{\alpha}+\frac{\beta}{\alpha+\beta} \frac{y}{\beta}\right)\right] \\
& \leq \frac{\alpha}{\alpha+\beta} \Phi\left[f \circ \frac{x}{\alpha}\right]+\frac{\beta}{\alpha+\beta} \Phi\left[f \circ \frac{y}{\beta}\right] \leq 1 .
\end{aligned}
$$

Therefore $\Phi_{f}(x+y) \leq a+\beta=\Phi_{f}(x)+\Phi_{f}(y)$ and (ii) is established. Finally, the fact that $\Phi$, satisfies property $(v)$ of symmetric norming functions follows directly from Weyl's theorem [13] which states that if $x_{0} y \in \mathbb{R} \sum_{n=1}^{k} x_{n} \leq \sum_{n=1}^{k} y_{n}, k=$ $1,2, \cdots$, and $g$ is a convex admissible function, then $\sum_{n=1}^{k} g\left(x_{n}\right) \leq \sum_{n=1}^{k} g\left(y_{n}\right)$, $k=1,2, \cdots$.

Theorem 5.4. Let $\Phi \in \mathcal{N}$ and $f \in \mathbb{C}_{c^{*}}$. Then $\mathcal{S}\left(f ; \mathcal{S}_{\Phi}\right)=\mathcal{S}_{\Phi_{f}}$ and $\mathcal{D}\left(f ; \mathcal{S}_{\Phi}^{0}\right)=\mathcal{S}_{\Phi^{*}}^{0}$

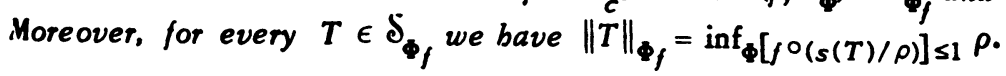

Proof. Let $x \in \in_{\Phi_{j}}$ Then it is not hard to check that

$$
\begin{aligned}
& \lim _{k \rightarrow \infty} \inf _{k} \rho \\
& =\inf \left\{\rho: \lim _{k \rightarrow \infty} \Phi\left[\sigma_{k}(f \circ(x / \rho))\right] \leq 1\right\}
\end{aligned}
$$

Therefore $\Phi_{f}(x)=\inf _{\Phi}[f \circ(x / \rho)] \leq 1 \rho$. This completes the proof of the last assertion of the theorem. Also, we deduce that $\mathfrak{E}_{\Phi_{f}} \subseteq \mathcal{E}\left(f ; \mathfrak{E}_{\Phi}\right)$. In order to prove the reverse inclusion we let $f^{\prime}$ be the right derivative of $f$ (which exists everywhere since $f$ is convex). From an elementary property of convex function, we observe that for every $\xi>0, f^{\prime}(\xi) \leq \xi^{-1}[f(2 \xi)-f(\xi)] \leq f^{\prime}(2 \xi)$ and hence $f(\xi / 2) \leq$ $1 / 2 \xi f^{\prime}(\xi / 2) \leq f(\xi)$. Now let $x \in \Subset\left(f ; G_{\Phi}\right)$. Then there exists $\alpha>0$ such that $f \circ(a x) \in \mathfrak{d}_{\phi}$. From the preceding observation we see that, for every $0<\delta \leq 1$, 


$$
\Phi\left[f \circ\left(\delta \frac{a}{2} x\right)\right] \leq \Phi\left[\left(\delta \frac{a}{2} x\right)\left(f^{\prime} \circ\left(\delta \frac{\alpha}{2} x\right)\right)\right] \leq \delta \Phi\left[\left(\frac{\alpha}{2} x\right)\left(f^{\prime} \circ\left(\frac{\alpha}{2} x\right)\right)\right] \leq \delta \Phi[f \circ(a x)] .
$$

Therefore, choosing $\delta$ so that $\delta \Phi[f \circ(a x)] \leq 1$ and setting $\alpha \delta / 2=1 / \rho$, we conclude that $\Phi(f \circ(x / \rho)) \leq 1$. It follows that $x \in \mathcal{E}_{\Phi_{f}}$, as desired. Finally, in order to prove the second statement of the theorem let $x \in D\left(f ; \mathcal{S}_{\Phi}^{0}\right)$. Then for every $a>0, \lim _{m \rightarrow \infty} \Phi\left[\tau_{m}(f \circ(a x))\right]=0$. For a given $\epsilon>0$ let $a=1 / \epsilon$. It follows that there exists $m_{0}$ such that, for every $m \geq m_{0}, \Phi\left[r_{m}(f \circ(x / \epsilon))\right] \leq 1$. Then $\inf _{\Phi}\left[\tau_{m}(f \circ(x / \rho))\right] \leq 1 \rho \leq \epsilon$, for every $m \geq m_{0}$. Thus, for every $m \geq m_{0}, \Phi_{f}\left[\tau_{m}(x)\right] \leq \epsilon$ and hence $x \in \mathfrak{S}_{\Phi_{j}}^{0}$. Conversely, suppose that $x \in \mathbb{G}_{\Phi_{f}}^{0}$ and let $a>0$ and $0<\epsilon<1$ be given. Then there exists $m_{1}$ such that for every $m \geq m_{1}$ we have $\Phi_{f}\left[\tau_{m}(x)\right] \leq$ $\epsilon / a$. Therefore, $1 \geq \Phi\left[r_{m}(f \circ(a x / \epsilon))\right] \geq \Phi\left[r_{m}(f \circ(a x))\right] / \epsilon$, for every $m \geq m_{1}$. From the last inequality we see that $x \in \mathscr{S}\left(f ; \mathfrak{C}_{\Phi}^{0}\right)$ and the theorem follows.

As in [10] we shall simply write $\delta(f)$ and $\mathscr{L}(f)$ in place of $\mathcal{S}\left(f ; \mathcal{C}_{1}\right)$ and $D\left(f ; C_{1}\right)$, for every $f \in \mathbb{Q}$.

Corollary 5.5. Let $f \in \mathbb{C}_{c}$. Then $S(f)$ is a maximal norm ideal and $\mathscr{D}(f)$ is the corresponding minimal norm ideal. In particular, $D(f)$ is the unique separable norm ideal which is a closed subspace of $S(f)$. Thus, $S(f)$ is separable if and only if $f$ is a mono-generating function.

Proof. It is a direct consequence of Remark 5.2, Theorem 5.4 and [5, Chapter 3, \$6].

Corollary 5.6. Let $f_{,} g \in \mathbb{C}_{c}$ and let $\Phi_{1}$ be the maximal symmetric norming function (see Remark 2.5). Then the following statements are equivalent:

(a) $\dot{S}(g) \subseteq \dot{S}(f)$.

(b) $\mathfrak{T}(g) \cong \mathscr{D}(f)$.

(c) $\mathscr{D}(g) \cong \mathscr{D}(f)$.

(d) $f<g$.

(e) $\left(\Phi_{1}\right)$ < $\left(\Phi_{1}\right)_{g^{\circ}}$

Proof. It follows immediately from Corollary 5.5, Theorem 2.3 and [10, Theorem 2.12].

The following theorem is a generalization of Theorem 2.8.

Theorem 5.7. Let $f \in \mathbb{C}_{c}$ and let $\Phi \in \boldsymbol{r}$ sucb that $\mathcal{S}_{\Phi} \subseteq \mathcal{C}$. Then $\mathcal{S}_{\Phi} \subsetneq$ $\mathcal{S}\left(f ; \mathcal{J}_{\phi}\right) \varsubsetneqq \mathcal{C}$ and bence $\mathcal{S}_{\Phi}^{0} \varsubsetneqq \mathcal{D}\left(f ; \mathcal{S}_{\Phi}^{0}\right)$.

Proof. The inclusion $\mathcal{S}\left(f ; \mathcal{J}_{\Phi}\right) \varsubsetneqq \mathcal{C}$ follows from the fact that $\mathcal{J}_{\Phi} \varsubsetneqq \mathcal{C}$ (Corollary 2.4) and [10, Theorem 2.22]. On the other hand, since $f(\xi) \leq \xi$, for every $0 \leq \xi \leq 1$, we see that $\dot{J}_{\Phi} \subseteq \dot{d}\left(f ; \dot{J}_{\Phi}\right)$. In order to prove the proper inclusion it suffices to show that $\sup _{x \in \mathfrak{\Im} ; x_{1} \neq 0}[\Phi(x) / \Phi,(x)]=\infty$. Let $a_{k}=1 / \Phi\left[\sigma_{k}(u)\right], k=$ $1,2, \ldots$ (where, as before $u_{n}=1, n=1,2, \ldots$ ). Then 


$$
\sup _{x \in \mathcal{B} ; x_{1} \neq 0}\left[\Phi(x) / \Phi_{f}(x)\right] \geq \sup _{k}\left\{\Phi\left[\sigma_{k}\left(a_{k} u\right)\right] / \Phi_{f}\left[\sigma_{k}\left(a_{k} u\right)\right]\right\}=\sup _{k} 1 / \Phi_{f}\left[\sigma_{k}\left(a_{k} u\right)\right] .
$$

Now we observe that $\Phi_{f}\left[\sigma_{k}\left(a_{k} u\right)\right]$ satisfies the identity

$$
1=\Phi\left[\sigma_{k}\left(f \circ \frac{a_{k}}{\Phi_{f}\left[\sigma_{k}\left(a_{k} u\right)\right]} u\right)\right]=\frac{1}{a_{k}} f\left(\frac{1}{\Phi_{f}\left[\sigma_{k}\left(a_{k} u\right)\right]}\right), \quad k=1,2, \ldots
$$

If $\boldsymbol{g}$ denotes the inverse function of $f$, then from the last formulas, we deduce that $\Phi_{f}\left[\sigma_{k}\left(\alpha_{k} u\right)\right]=a_{k} / g\left(a_{k}\right), k=1,2, \ldots$. Since $f \in \mathbb{Q}_{c}$, it satisfies (**) and hence $\lim _{\xi \rightarrow 0} g(\xi) / \xi=\infty$. Also, we recall that $\inf _{k} a_{k}=1 / \sup _{k} \Phi\left[\sigma_{k}(u)\right]=0$ (because $\dot{S}_{\phi} \subsetneq \mathcal{C}$ ). Therefore

$$
\sup _{x \in \delta_{;} x_{1} \neq 0}\left[\Phi(x) / \Phi_{f}(x)\right] \geq \sup _{k} 1 / \Phi_{f}\left[\sigma_{k}\left(a_{k} u\right)\right]=\sup _{k} g\left(a_{k}\right) / a_{k}=\infty .
$$

Theorem 5.8. Let $f \in \mathbb{U}_{c}$ and $\Phi \in \boldsymbol{r}$. Then the ideals $\mathcal{S}\left(f ; \mathcal{J}_{\Phi}^{0}\right)$ and $\mathscr{L}\left(f ; \mathcal{J}_{\Phi}\right)$ are closed subspaces of $\delta\left(g ; \dot{J}_{\Phi}\right)$, and bence they are (perbaps trivial) intermediate symmetric norm ideals between the minimal norm ideal $\mathscr{D}\left(f ; \mathcal{S}_{\Phi}^{0}\right)$ and the maximal norm ideal $\dot{d}\left(f ; \dot{J}_{\Phi}\right)$ corresponding to the symmetric norming function $\Phi_{f}$

Proof. We show first that $\delta\left(f ; S^{0}\right)$ is closed. To this end, let $\left\{T_{n}\right\}$ be a sequence in $S\left(f ; \mathcal{S}_{\Phi}\right)$ and let $T \in S\left(f ; \mathcal{S}_{\Phi}\right)$ such that $\lim _{n \rightarrow \infty}\left\|T-T_{n}\right\|_{\Phi_{f}}=0$. For an arbitrary positive number $\epsilon$ less than one choose a positive integer $k$ such that $\left\|T-K_{k}\right\|_{\Phi_{f}} \leq \epsilon$. Since $T_{k} \in S\left(f ; \delta_{\Phi}^{0}\right)$, there exist $0<a<1 / 2$ and $m_{0}$ such that $\Phi\left[r_{m}\left(f \circ s\left(2 \alpha T_{k}\right)\right)\right]$ $<\epsilon$, for every $m \geq m_{0}$. Since $1 / \epsilon>1$ we also have $1 \geq \Phi\left[f \circ s\left(\left(T-T_{k}\right) / \epsilon\right)\right] \geq$ $\Phi\left[f \circ s\left(T-T_{k}\right)\right] / \epsilon$. From Fan's inequality [3] we see that

$$
\sum_{n=1}^{j} s_{n}\left[\left(T-T_{k}\right)+T_{k}\right] \leq \sum_{n=1}^{j}\left[s_{n}\left(T-T_{k}\right)+s_{n}\left(T_{k}\right)\right]
$$

and therefore

$$
\begin{aligned}
\Phi\left[\tau_{m}(f \circ s(\alpha T))\right] & \leq \Phi\left[r_{m}\left(f \circ\left[s\left(\alpha\left(T-T_{k}\right)\right)+s\left(\alpha T_{k}\right)\right]\right)\right] \\
& \leq 1 / 2 \Phi\left[r_{m}\left(f \circ s\left(2 \alpha\left(T-T_{k}\right)\right)\right)\right]+1 / 2 \Phi\left[\tau_{m}\left(f \circ s\left(2 \alpha T_{k}\right)\right)\right] \\
& \leq 1 / 2 \Phi\left[f \circ s\left(T-T_{k}\right)\right]+1 / 2 \Phi\left[r_{m}\left(f \circ s\left(2 \alpha T_{k}\right)\right)\right]<\epsilon / 2+\epsilon / 2=\epsilon,
\end{aligned}
$$

for every $m \geq m_{0}$. Thus, $T \in \dot{\mathcal{S}}\left(f ; \dot{\mathcal{J}}_{\Phi}^{0}\right)$ as desired. In order to show that $\mathscr{D}\left(f ; \dot{J}_{\phi}\right)$ is closed, let $\left\{S_{n}\right\}$ be a sequence in $\mathcal{D}\left(f ; \dot{J}_{\Phi}\right)$ such that $\lim _{n \rightarrow \infty}\left\|S-S_{n}\right\|_{\Phi_{f}}=0$, for some $S \in \dot{S}\left(f ; \dot{J}_{\Phi}\right)$. For a given $a>0$, let $j$ be a positive integer such that $\left\|S-S_{j}\right\|_{\Phi_{f}}<1 / 2 a$. It follows (using again Fan's inequality) that

$$
\begin{aligned}
\Phi[f \circ s(\alpha s)] \leq & \Phi\left[f \circ\left[s\left(\alpha\left(s-s_{j}\right)\right)+s\left(\alpha s_{j}\right)\right]\right] \\
\leq & 1 / 2 \Phi\left[f \circ s\left(2 \alpha\left(s-s_{j}\right)\right)\right] \\
& +1 / 2 \Phi\left[f \circ s\left(2 \alpha S_{j}\right)\right]<1 / 2+1 / 2 \Phi\left[f \circ s\left(2 \alpha s_{j}\right)\right] .
\end{aligned}
$$


Since $S_{j} \in \mathscr{D}\left(f ; \dot{J}_{\Phi}\right)$ we conclude that $S \in \mathscr{D}\left(f ; \dot{J}_{\Phi}\right)$ and the proof of the theorem is completed.

Remark 5.9. Let $f \in \mathbb{Q}_{c}$ and $\Phi \in \pi$. The intermediate ideals $\delta\left(f ; \dot{S}_{\Phi}^{0}\right)$ and $\mathscr{D}\left(f ; \partial_{\Phi}\right)$ might coincide with either $\mathcal{J}\left(f ; \partial_{\Phi}\right)$ or $\mathscr{D}\left(f ; \mathcal{S}_{\Phi}^{0}\right)$. In fact, as we observed in Remark 5.2, if $f$ is a mono-generating function, then $\mathscr{S}\left(f ; S_{\Phi}\right)=\mathscr{L}\left(f ; S_{\Phi}\right)$ and $\mathcal{S}\left(f ; \mathcal{S}_{\Phi}^{0}\right)=\mathscr{I}\left(f ; \mathcal{S}_{\Phi}^{0}\right)$. On the other hand, let $b \in \mathbb{Q}_{c}$ such that $b(\xi)=e^{-1 / \xi}$, $0<\xi<1 / 2$. It can be proved that $\mathscr{T}(b ; \mathscr{L}(b))=\mathscr{L}^{c}(b ; \mathcal{S}(b)) \varsubsetneqq \mathcal{S}(b ; \mathscr{D}(b))=\mathcal{S}(b ; \mathcal{S}(b))$.

An interesting situation, with regard to the intermediate symmetric norm ideals mentioned above, occurs when $\mathcal{S}_{\Phi}=\mathcal{S}_{\Phi}^{0}=\mathcal{C}_{1}$. In this connection it is natural to ask:

Let $f \in \mathbb{Q}_{c}$ be a non mono-generating function. Does there exist a nontrivial intermediate symmetric norm ideal between $\mathscr{T}(f)$ and $\mathcal{S}(f)$ ?

We suggest the following approach to attack this problem. Let us suppose that $f$ can be expressed as $f(\xi)=f_{1}\left[f_{2}(\xi)\right], \xi \geq 0$, where $f_{1}, f_{2} \in \mathbb{Q}_{c}$ and $f_{1}, f_{2}$ are not mono-generating functions. Since $\delta(f)=\delta\left(f_{2} ; \delta\left(f_{1}\right)\right)$ and $\mathscr{L}(f)=$ $\mathscr{I}\left(f_{2} ; \mathscr{L}\left(f_{1}\right)\right)$, the above problem would be solved for the function $f$ if one of the ideals $\mathscr{S}\left(f_{2} ; \mathfrak{I}\left(f_{1}\right)\right)$ or $\mathscr{I}\left(f_{2} ; \mathscr{S}\left(f_{1}\right)\right)$ properly contains $\mathscr{L}\left(f_{2} ; \mathfrak{I}\left(f_{1}\right)\right)$ and is strictly smaller than $\mathcal{S}\left(f_{2} ; \mathcal{S}\left(f_{1}\right)\right)$. However, this method might not be successful because of the example with the function $f$ presented above.

The existence of nontrivial intermediate symmetric norm ideals was proved by Mitjagin in [8]. To prove his result Mitjagin used the nonseparable maximal norm ideals introduced by Gohberg and KreYn in [6] (they are of the form $(T)^{\times \times}\left\langle\mathcal{C}_{1}\right\rangle, T \in \mathcal{C}-\mathcal{C}_{1}$ of Remark $4.7(\mathrm{e})$. These ideals are essentially different from the ideals $S(f)$ and $\mathscr{T}(f), f \in \mathbb{Q}_{c}$.

6. Conjugate ideals of the ideals $S\left(f ; \mathcal{S}_{\Phi}\right)$ and $\mathscr{I}\left(f ; \mathcal{S}_{\Phi}^{0}\right)$. We start this section by recalling some facts about convex functions [14].

Let $f \in \mathbb{Q}_{c}$, and let $f^{\prime}$ denote the right derivative of $f$. For each $\xi \geq 0$ let $g^{\prime}(\xi)=\sup _{\eta} f^{\prime}(\eta) \leq \xi$. Since $f$ satisfies condition $(* * *), \lim _{\xi \rightarrow \infty} f^{\prime}(\xi)=\infty$ and hence $g^{\prime}$ is a nonnegative function defined on the whole nonnegative real line. Furthermore, the fact that $f$ satisfies condition (**) guarantees that $f^{\prime}$ and then $\boldsymbol{g}^{\prime}$ are in $\mathcal{G}$. It can be proved that

$$
f^{\prime}\left[g^{\prime}(\xi)\right] \geq \xi, \quad g^{\prime}\left[f^{\prime}(\xi)\right] \geq \xi,
$$

for every $\xi \geq 0$, and

$$
f^{\prime}\left[g^{\prime}(\xi)-\epsilon\right] \leq \xi, \quad g^{\prime}\left[f^{\prime}(\eta)-\delta\right] \leq \eta,
$$

for every $\xi>0, \eta>0$ and every $\epsilon, \delta$ such that $0<\epsilon<g^{\prime}(\xi), 0<\delta<f^{\prime}(\eta)$. Thus, if $f^{\prime}$ is continuous, then $g^{\prime}$ is the inverse function of $f^{\prime}$. In the standard terminology, the function $g^{\prime}$ is called the right inverse of $f^{\prime}$ and the function $g$ defined by

$$
g(\xi)=\int_{0}^{\xi} g^{\prime}(\theta) d \theta
$$


is called the conjugate of $f$ in the sense of Young. It follows that $g / g(1) \in \mathbb{C}_{c}$ and that the conjugate of $g$ in the sense of Young is the function $f$. The following is the well-known Young inequality:

$$
\xi \eta \leq f(\xi)+g(\eta), \quad \xi, \eta \geq 0 .
$$

Equality holds whenever either $\xi=g^{\prime}(\eta)$ or $\eta=f^{\prime}(\xi)$.

Using the techniques provided by the theory of Orlicz spaces [14, Chapter 5] we generalize, in this section, Theorem 4.5 by proving that if $f$ and $g$ are conjugate in the sense of Young, then $\mathcal{S}\left(f ; \mathcal{S}_{\Phi}\right)$ and $\mathcal{S}\left(g ; \mathcal{S}_{\Phi}\right)$ are $\mathcal{S}_{\Phi}$-conjugate one of each other, for every $\Phi \in \Re$. The following two lemmas are central to our purposes.

Lemma 6.1. Let $f \in \mathfrak{Q}_{c}$ and let $g$ be the conjugate function of $f$ in the sense of Young. Then $g(1)<1$.

Proof. We know that $f^{\prime}(1)=f(1)+g\left(f^{\prime}(1)\right)$. Since $f(1)=1$ and $g\left(f^{\prime}(1)\right)>0$ we deduce that $f^{\prime}(1)>1$. Let $0<\epsilon<f^{\prime}(1)-1$. Then $1<f^{\prime}(1)-\epsilon$ and hence $g^{\prime}(1) \leq g^{\prime}\left(f^{\prime}(1)-\epsilon\right) \leq 1$. Since $g^{\prime} \in \mathbb{Q}$ and $g^{\prime}(1) \leq 1$ we finally conclude that $g(1)=\int_{0}^{1} g^{\prime}(\theta) d \theta<1$, as desired.

Lemma 6.2. Let $f \in \mathbb{Q}_{c}, \Phi \in M$ and let $f^{*}=g / g(1)$, where $g$ is the conjugate of $f$ in the sense of Young. Then for every $x \in \mathfrak{F}$ we bave

$$
g(1) \Phi_{f^{*}}(x) \leq\left(\Phi_{f}\right)_{\phi}^{*}(x) \leq[1+g(1)] \Phi_{f^{*}}(x) .
$$

Proof. From Young's inequality we see that for every $x, y \in \mathfrak{F}, x_{1} \neq 0$, $y_{1} \neq 0$, we have

$$
\begin{aligned}
& \Phi\left[\left(x / \Phi_{f^{*}}(x)\right)\left(y / \Phi_{f}(y)\right)\right] \leq \Phi\left[g \circ\left(x / \Phi_{f^{*}}(x)\right)+f \circ y / \Phi_{f}(y)\right] \\
& \leq g(1) \Phi\left[f^{*} \circ\left(x / \Phi_{f^{*}}(x)\right)\right]+\Phi\left[f \circ\left(y / \Phi_{f}(y)\right)\right] \leq g(1)+1 .
\end{aligned}
$$

Therefore, for every $x, y \in \mathfrak{F}, \Phi(x y) \leq\left[1+g^{(1)}\right] \Phi_{f}(x) \Phi_{f}(y)$, and hence, $\left(\Phi_{f}\right)_{\Phi}^{*}(x) \leq[1+g(1)] \Phi_{f}^{*}(x)$. This completes the proof of the second inequality. The proof of the first inequality will be given in several steps. We first observe (using Lemma 5.3) that $\{y \in \mathfrak{F}: \Phi(y) \leq 1\}=\{y \in \mathfrak{F}: \Phi(f \circ y) \leq 1\}$. Now let $x \in \mathfrak{F}$, $x_{1} \neq 0$, and define $z=g(1) x /\left(\Phi_{f}\right)_{\Phi}^{*}(x)$. Clearly, it suffices to prove that $\Phi_{f} z \leq 1$. Since

$$
\Phi\left(f^{*} \circ z\right)=\frac{1}{g(1)} \Phi(g \circ z) \leq \frac{1}{g(1)} \Phi\left[g \circ z+f \circ\left(g^{\prime} \circ z\right)\right]=\frac{1}{g(1)} \Phi\left[z\left(g^{\prime} \circ z\right)\right],
$$

we need only to show that $\Phi\left[z\left(g^{\prime} \circ z\right)\right] / g(1) \leq 1$. On the other hand, the validity of the last inequality follows from the validity of $\Phi\left[f \circ\left(g^{\prime} \circ z\right)\right] \leq 1$. In fact, if the last inequality holds, we deduce that 


$$
\left.\frac{1}{g(1)} \Phi\left[z\left(g^{\prime} \circ z\right)\right] \leq \sup _{y \in \mathcal{J} ; \Phi(f \circ y) \leq 1} \frac{\Phi(z y)}{g(1)}=\frac{1}{g(1)}(\Phi)\right)_{\Phi}^{*}(z)=1 .
$$

Thus in order to complete the proof of the theorem it remains to show $\Phi\left[f \circ\left(g^{\prime} \circ z\right)\right]$ $\leq 1$. If, on the contrary, $\Phi\left[f \circ\left(g^{\prime} \circ z\right)\right]>1$ we let $w=\left(g^{\prime} \circ z\right) / \Phi\left[f \circ\left(g^{\prime} \circ z\right)\right]$. Then, for every $n=1,2, \cdots, f\left(w_{n}\right) \leq f\left(g^{\prime}\left(z_{n}\right)\right) / \Phi\left[f \circ\left(g^{\prime} \circ z\right)\right]$, and hence $\Phi(f \circ w) \leq 1$. Now, we observe that $\Phi[z w / g(1)] \leq\left(\Phi_{f}^{n}\right)_{\Phi}^{*}[z / g(1)] \Phi(w) \leq 1$ and hence $\Phi\left[f \circ\left(g^{\prime} \circ z\right)\right]$ $\leq \Phi\left[f \circ\left(g^{\prime} \circ z\right)+g \circ z\right]=\Phi\left[z\left(g^{\prime} \circ z\right)\right]=g(1) \Phi\left[f \circ\left(g^{\prime} \circ z\right)\right]=\Phi[z w / g(1)] \leq$ $g(1) \Phi\left[f \circ\left(g^{\prime} \circ z\right)\right]$. Since $g(1)<1$ (Lemma 6.1) and $z_{1} \neq 0$ (because $x_{1} \neq 0$ ), the last chain of inequalities gives the desired contradiction.

Theorem 6.3. Let $f \in \mathbb{Q}_{c}$ and let $g$ be the conjugate of $f$ in the sense of Young. Then for every $\Phi \in \Re^{c}$, it follows that $\delta\left(f ; \mathbb{S}_{\Phi}\right)$ and $\mathcal{S}\left(g ; \mathcal{S}_{\Phi}\right)$ are $\mathcal{S}_{\Phi}$-conjugate one of each other. Moreover, the $S_{\Phi}^{0}$-conjugate and the $\delta_{\phi}$-conjugate of $\mathcal{I}\left(f ; \mathcal{S}_{\Phi}^{0}\right)$ coincides with $\mathcal{S}\left(g ; \delta_{\phi}\right)$.

Proof. It is a direct consequence of Theorem 4.3, Theorem 5.4 and Lemma 6.2.

Corollary 6.4. Let $f \in \mathbb{Q}_{c}$ and let $g$ be the conjugate of $f$ in the sense of Young. Then $S(f)$ and $S(g)$ are Kötbe-dual one of each other. Moreover, $S(g)$ is the topological dual and the Köthe-dual of $\mathcal{L}(f)$. Also, $S(g)$ can be embedded, isomorpbically and topologically, in the topological dual of $\mathcal{S}(f)$.

Proof. It follows immediately from Remark 4.7(c) and Theorem 6.3.

Theorem 6.5. Let $f \in \mathbb{Q}_{c}$ and let $g$ be the conjugate of $f$ in the sense of Young. The following statements are equivalent:

(a) $\mathscr{L}(f)$ is reflexive,

(b) $S(f)$ is reflexive,

(c) $\mathscr{L}(g)$ is reflexive,

(d) $S(g)$ is reflexive,

(e) $f$ and $g$ are mono-generating functions.

Proof. We recall that a Banach space is reflexive if and only if its topological dual is reflexive. Therefore, if $\mathscr{T}(f)$ is reflexive, it follows that $\mathscr{T}(f)=\mathscr{S}(f)$ and $\mathscr{L}(g)=S(g)$. Thus, (a) implies (b), (c), (d) and (e). Similarly, (c) implies (a), (b), (d) and (e). Since $\delta(f)$ is the topological dual of $\mathscr{L}(g)$, then if $S(f)$ is reflexive so is $\mathscr{T}(g)$; analogously, if $\mathscr{S}(g)$ is reflexive so is $\mathscr{L}(f)$. On the other hand, since (e) implies clearly (a), (b), (c), and (d) the proof is completed.

Remark 6.6. (a) As before, let $f_{(p)}$ be the function in $\mathcal{Q}_{c}, p \geq 1$ defined by $f_{(p)}(\xi)=\xi^{p}, \xi \geq 0$. The conjugate of $f_{(p)}$ in the sense of Young is the function $f_{(q)} /\left(1+p^{q-1}\right), 1 / p+1 / q=1$. Thus, Theorem 6.3 is the natural generalization of Theorem 4.5.

(b) Let $f_{1}, f_{2} \in \mathbb{C}_{c}$, and let $g_{1}, g_{2}$ be the conjugate functions in the sense 
of Young of $f_{1}, f_{2}$ respectively. It follows that $f_{1} \prec f_{2}$ if and only if $g_{2} \prec g_{1}$ and hence $f_{1} \sim f_{2}$ if and only if $g_{1} \sim g_{2}$. In view of this observation, to determine the Köthe-dual of $S(f), f \in Q_{c}$ it suffices to find the conjugate in the sense of Young of a function in $\hat{Q}_{c}$ equivalent to $f$. We illustrate this remark with an interesting example. Let $b$ be the function in $Q_{c}$ of Remark 5.7 (i.e., $b$ satisfies $b(\xi)=e^{-1 / \xi}, 0<\xi<1 / 2$ ). Also let $b_{j}, 1 \leq j \leq 4$, be functions in $\mathbb{Q}_{c}$ satisfying $b_{1}(\xi)=\xi b(\xi), b_{2}(\xi)=\int_{0}^{\xi} b(\theta) d \theta, b_{3}(\xi)=$ $-1 / \ln \xi, b_{4}(\xi)=\int_{0}^{\xi} b_{3}(\theta) d \theta, 0<\xi<1 / 2$. It is easy to check that $b \sim b_{1}$ and [10, Theorem 3.4] $b_{1} \sim b_{2}$. Since $b_{2}^{\prime}=b$ and the inverse of $b$ is equivalent to $b_{3}$, the conjugate of $b$ in the sense of Young is equivalent to $b_{4}$. Using again [10, Theorem 3.4] we conclude that the Köthe-dual of $S(b)$ is $\delta\left(b_{s}\right)$, where $b_{s} \in \mathbb{Q}_{c}$ satisfies $b_{s}(\xi)=-\xi / \ln \xi, 0<\xi<1 / 2$.

(c) In parts (a) and (b) one of the conjugate functions considered is monogenerating. With the following example we show that this might not happen in general. Let $\lambda_{n}=1 / n !, n=1,2, \cdots$, and let $f^{\prime} \in \mathcal{U}^{\prime}$ such that $\lim _{\xi \rightarrow \infty} f^{\prime}(\xi)=\infty$ and $f^{\prime}(\xi)=\lambda_{n}, \lambda_{n+1} \leq \xi<\lambda_{n}, n=1,2, \ldots$. Then the right inverse $g^{\prime}$ of $f^{\prime}$ satisfies: $g^{\prime} \in \mathbb{C}^{n+1}$ and $g^{\prime}(\xi)=\lambda_{n+1}, \lambda_{n+1} \leq \xi<\lambda_{n}, n=1,2, \ldots$ Let $1 . g \in \mathbb{Q}$ such that $f(\xi)=\int_{0}^{\xi} f^{\prime}(\theta) d \theta, g(\xi)=\int_{0}^{\xi^{n}} g^{\prime}(\theta) d \theta$. It follows that $f$ and $g$ are conjugate in the sense of Young and neither $f$ nor $g$ is a mono-generating function. In fact,

$$
\begin{aligned}
n f\left(\lambda_{n}\right) & =n \int_{0}^{\lambda_{n}} f^{\prime}(\theta) d \theta \leq n \int_{0}^{\lambda_{n}} \lambda_{n} d \theta=\lambda_{n-1} \lambda_{n}=\int_{\lambda_{n}}^{2 \lambda_{n}} \lambda_{n-1} d \theta=\int_{\lambda_{n}}^{2 \lambda_{n}} f^{\prime}(\theta) d \theta \\
& \leq \int_{0}^{2 \lambda_{n}} f^{\prime}(\theta) d \theta=f\left(2 \lambda_{n}\right), \quad n=2,3, \ldots
\end{aligned}
$$

A similar calculation shows that $(n+1)_{g}\left(\lambda_{n}\right) \leq g\left(2 \lambda_{n}\right), n=1,2, \ldots$ Therefore $f$ and $g$ are not mono-generating functions, as asserted.

7. Some problems on joins and intersections of ideals. In this section we study the following questions.

(a) Let $\Phi \in \pi$ such that $\delta_{\Phi} \subseteq \mathcal{C}$ and let $\mathcal{G}_{k}, k=1,2, \cdots$, be a countable family of ideals strictly larger than $\delta_{\Phi}$. Does $\bigcap_{k=1}^{\infty} g_{k}$ properly contain $\delta_{\Phi}$ ?

$(\beta)$ Let $\Phi \in \Re$ and let $g_{k}, k=1,2, \cdots$, be a countable family of ideals properly contained in $\delta_{\Phi}^{0}$. Is the join $\bigvee_{k=1}^{\infty} g_{k}$ strictly included in $\delta_{\Phi}^{0}$ ?

Clearly, problems $(\alpha)$ and $(\beta)$ can be split into the following ones.

$\left(\alpha_{1}\right)$ Let $\Phi \in \boldsymbol{r}$ such that $\mathcal{S}_{\Phi} \subseteq \mathcal{C}$ and let $\mathcal{G}_{k}, k=1,2, \ldots$, be a decreasing countable family of ideals such that $S_{\Phi} \subsetneq g_{k}, k=1,2, \ldots$. Is it true that $\delta_{\Phi} \subsetneq \bigcap_{k=1}^{\infty} g_{k}$ ?

$\left(a_{2}\right)$ Let $\Phi \in r$ such that $\mathcal{S}_{\Phi} \subseteq \mathcal{C}$ and let $g, g$ be two ideals such that $\delta_{\Phi} \subsetneq 9, \delta_{\Phi} \subsetneq \mathcal{J}$. Does it follow that $\delta_{\Phi} \varsubsetneqq g \cap \mathcal{J}$ ? 
$\left(\beta_{1}\right)$ Let $\Phi \in r$ and let $g_{k}, k=1,2, \ldots$, be an increasing countable family of ideals such that $g_{k} \subsetneq \delta_{\Phi}^{0}, k=1,2, \ldots$ Is it true that $\bigcup_{k=1}^{\infty} g_{k} \subsetneq \delta_{\Phi}^{0}$ ?

$\left(\beta_{2}\right)$ Let $\Phi \in \pi$ and let $g, g$ be two ideals such that $g \cong \delta_{\phi}^{0}, \mathcal{J} \varsubsetneqq \delta_{\phi}^{0}$. Does it follow that $g \vee \mathcal{J} \varsubsetneqq \mathcal{S}_{\Phi}^{0}$ ?

Problems $\left(\alpha_{2}\right)$ and $\left(\beta_{2}\right)$ in the case $\delta_{\Phi}=\delta_{\Phi}^{0}=C_{p}, p \geq 1$, have negative answers (see [7, Remark 4.5]). Problem $(\beta)$ for the case $\delta_{\phi}^{0}=\mathcal{C}$ was posed in $[1, \$ 6]$. In the present section we solve problem $\left(\alpha_{2}\right)$ in the negative and problem $\left(\beta_{1}\right)$ in the affirmative. Also, we give partial answers to questions $\left(\alpha_{1}\right)$ and $\left(\beta_{2}\right)$.

In what follows, for the sake of brevity, we shall simply write $\sqrt{ }$ in place of $\Lambda(\mathcal{C})$. The set of all nondecreasing sequences of nonnegative real numbers tending to $\infty$ will be denoted by $\Gamma$, and the elements of $\Gamma$ by $b, c, d$, etc.

To begin with let us establish the following criterion which is a generalization of a result given in $[10, \$ 4]$.

Theorem 7.1. Let $f \in \mathcal{Q}$, and let $\Phi \in \boldsymbol{r}$ sucb that $\mathcal{S}_{\phi} \subseteq \mathcal{C}$. Then

(a) $x \in \mathscr{P}\left(f ; \mathcal{G}_{\Phi}^{0}\right)$ if and only if there exists $c \in \Gamma$ such that $f \circ(c x) \in \mathcal{G}_{\Phi}^{0}$,

(b) for $y \in \Omega$, we bave $y \notin \mathcal{G}\left(f ; \mathcal{G}_{\uparrow}\right)$ if and only if there exists $t \in \mathcal{G}$ sucb that $f \circ(t y) \notin \tilde{E}_{\phi}$.

Proof. (a) Let $x \in \mathscr{Q}\left(f ; \mathfrak{S}_{\phi}^{0}\right)$. Then, by deleting the first few terms of the sequence $x$, we can assume, without loss of generality, that $\Phi(f \circ x) \leq 1$. We define the sequence $\left\{m_{j}\right\}$ of positive integers as follows: let $m_{1}$ be the first positive integer such that $\Phi\left[r_{m_{1}}(f \circ 2 x)\right] \leq 1 / 2$. Having defined $m_{j}, j \geq 1$, let $m_{j+1}$ be the first positive integer such that $m_{j+1}>m_{j}$, and $\Phi\left[\tau_{m_{j+1}}(f \circ(j+2) x)\right] \leq$ $1 / 2^{j+1}$. Now let $c \in \Gamma$ given by $c_{n}=1,1 \leq n \leq m_{1}, \cdots, c_{n}=j+1, m_{j}+1 \leq$ $n \leq m_{j+1}$. We assert that $f \circ(c x) \in \mathcal{E}_{\phi}^{0}$. In fact, let $\epsilon>0$ be given and let $j$ be sufficiently large so that $1 / 2^{j}<\epsilon / 2$, then

$$
\begin{aligned}
\Phi\left[r_{m_{j}}(f \circ(c x))\right] & =\lim _{b \rightarrow \infty} \Phi\left[\sigma_{m_{b}}\left(r_{m_{j}}[f \circ(c x)]\right)\right] \leq \lim _{b \rightarrow \infty} \sum_{i=j}^{b} \Phi\left[\sigma_{m_{i+1}}\left(r_{m_{i}}[f \circ(i+1) x]\right)\right] \\
& \leq \lim _{b \rightarrow \infty} \sum_{i=j}^{b} \frac{1}{2^{i}}=\frac{1}{2^{j-1}}<\epsilon .
\end{aligned}
$$

Thus, $f \circ(c x) \in \mathbb{G}_{\Phi}^{0}$, as asserted. Since the reverse implication of part (a) can be easily seen to be valid not only for $€_{\$}^{0}$ but also for every characteristic set, the proof of part (a) is finished.

(b) Let $y \in \mathfrak{R}$. If there exists $t \in \mathbb{E}$ such that $f \circ(t y) \notin \mathbb{E}$, it readily follows that $y \notin \mathcal{G}\left(f ; \Xi_{\Phi}\right)$. Conversely, assume that $y \notin \mathcal{G}\left(f ; \mathcal{G}_{\phi}\right)$. Let us define the sequence $\left\{b_{n}\right\}$ of positive integers as follows: $b_{1}$ is the first positive integer such that $\Phi\left[\sigma_{b_{1}}(f \circ y)\right] \geq 1$; having defined $b_{j}, j \geq 1$, let $b_{j+1}$ be the first positive integer such that $b_{j+1} \geq b_{j}, \Phi\left(\sigma_{b_{j+1}}\left[r_{b_{j}}(f \circ y /(j+1))\right]\right) \geq j+1$. Now, let $t \in \mathbb{S}$ 
be given by $t_{n}=1,1 \leq n \leq b_{1}, \cdots, t_{n}=1 /(j+1), b_{j}+1 \leq n \leq b_{j+1}$. We claim that $f \circ(t y) \notin E_{\Phi}$. In fact,

$$
\begin{aligned}
\lim _{k \rightarrow \infty} \Phi\left(\sigma_{k}[f \circ(t y)]\right) & =\lim _{j \rightarrow \infty} \Phi\left(\sigma_{b_{j+1}}[f \circ(t y)]\right) \\
& \geq \lim _{j \rightarrow \infty} \Phi\left(\sigma_{b_{j+1}}\left[r_{b_{j}}(f \circ y /(j+1))\right]\right) \geq \lim _{j \rightarrow \infty}(j+1)=\infty .
\end{aligned}
$$

Therefore, $f \circ(t y) \notin \epsilon_{\phi}$ and our claim is proved.

As observed in [10, Remark 4.5], if $f, g \in \mathbb{Q}$ and $\mathcal{J}$ is any ideal of $\mathscr{L}$, then $\mathcal{S}(f \vee g ; \mathcal{I})=\mathcal{S}(f ; \mathcal{J}) \cap \mathcal{S}(g ; \mathcal{I}), \mathscr{I}(f \vee g ; \mathcal{I})=\mathscr{L}(f ; \mathcal{I}) \cap \mathcal{I}(g ; \mathcal{I}), \mathcal{S}(f \wedge g ; \mathcal{J})=\mathcal{S}(f ; \mathcal{J})$ $\vee \mathcal{S}(g ; \mathcal{J})$. The remaining lattice property for the ideals of the form $\mathscr{I}(f ; \mathcal{J})$ is not known to be true in general, In [10, Theorem 4.7] it is shown that that property holds for $I=C_{1}$. By employing an argument identical to the one used in the proof of [10, Theorem 4.8], together with Lemma 7.1 (a) the following generalization can be obtained.

Theorem 7.2. Let $f, g \in \mathbb{Q}$ and let 9 be a minimal norm ideal. Tben $\mathscr{I}(f \wedge g ; \mathfrak{I})=\mathscr{L}(f ; \mathcal{I}) \vee \mathscr{D}(g ; \mathcal{g})$.

In the next theorem we give a partial answer to problem $\left(\beta_{2}\right)$ for the case $\delta_{\Phi}^{0}=\mathcal{C}$.

Theorem 7.3. Let $f \in \mathbb{C}$, let $g$ be a norm ideal such that $\mathcal{S}(f ; g) \subseteq \mathcal{C}$ and let $g$ be another ideal of $\mathcal{L}$ sucb that $g \subseteq \mathcal{C}$. Then $g \vee S(f ; g) \varsubsetneqq \mathcal{C}$.

Proof. Since $S(f ; g) \subseteq \mathcal{C}$, it follows $[10$, Theorem 2.22] that $g \subseteq \mathcal{C}$. Then (Theorems 2.1 and 2.3 ) there exists $\Psi \in \boldsymbol{\gamma}$ such that $g \subseteq \delta_{\Psi} \subseteq \mathcal{C}$. Thus, it suffices to prove that $g \vee S\left(f ; \delta_{\psi}\right) \subseteq \mathcal{C}$. Let $w \in \mathcal{E}$ such that $w \notin \Lambda(\mathcal{J})$. Since $\delta_{\downarrow} \subseteq \mathcal{S} \lim _{k \rightarrow \infty} \Psi\left[\sigma_{k}(u)\right]=\infty$ (as usual $u_{n}=1, n=1,2, \ldots$ ). And hence for each $m=1,2, \ldots$ there exists a positive integer $k_{m}$ such that $\Psi\left[\sigma_{k_{m}}(u)\right] \geq m / f\left(w_{m} / m\right)$. Let $z$ be the sequence defined by $z_{n}=w_{1}, 1 \leq n \leq k_{1}, \cdots, z_{n}=w_{j}, 1+\sum_{i=1}^{j} k_{i}$ $\leq n \leq \Sigma_{i=1}^{j+1} k_{i}$. Since $z \in \mathbb{E}$, the proof of the theorem will be completed after showing that $z \notin \Lambda(\mathcal{J}) \vee \mathrm{G}\left(f ; \mathfrak{E}_{\Psi}\right)$. Suppose that $z \in \Lambda(\mathcal{g}) \vee \mathrm{G}\left(f ; \mathcal{G}_{\psi}\right)$. Then there must exist $x \in \Lambda(g), y \in G\left(f ; \mathcal{G}_{\psi}\right)$ such that $z=x \vee y$. If for some $j$, the element $w_{j}$ does not appear in the sequence $x$, it has to appear $k_{j}$ times in $y$. If all but a finite number of terms of the sequence $w$ appeared in $x$ it would follow that $w \in \Lambda(\mathscr{J})$, contradicting the definition of the sequence $w$. Thus, there must exist a subsequence of $w$ whose elements $w_{n_{j}}$ appear in $y$ together with all its $k_{n_{j}}$ repetitions. Let $z^{\prime}$ be the subsequence of $z$ defined by $z_{n}^{\prime}=w_{n 1}, 1 \leq n \leq k_{n_{1}}, \ldots$, $z_{n}^{\prime}=w_{n_{j}}, 1+\sum_{i=1}^{j} k_{n_{i}} \leq n \leq \sum_{i=1}^{j+1} k_{n_{i}}$. Also let $t \in \Gamma_{\text {given by } t_{n}=1 / n_{1}, 1 \leq}^{n_{1}}$ $n \leq n_{1}, \cdots, t_{n}=1 / n_{j}, 1+\sum_{i=1}^{j} k_{n_{j}}^{n_{i}} \leq n \leq \sum_{i=1}^{j+1} k_{n_{i}}$. Since $z^{\prime}$ is a subsequence of $y$, it follows that $z^{\prime} \in G\left(f ; \tilde{S}_{\psi}\right)^{\prime}$. On the other hand, 


$$
\lim _{m \rightarrow \infty} \Psi\left[\sigma_{m}\left(f \circ t z^{\prime}\right)\right] \geq \lim _{j \rightarrow \infty} f\left(w_{n_{j}} / n_{j}\right) \Psi\left[\sigma_{n_{j}}(u)\right] \geq \lim _{j \rightarrow \infty} n_{j}=\infty
$$

and therefore $f \circ\left(t z^{\prime}\right) \notin \mathfrak{E}_{\phi}$. Since $z^{\prime} \in \mathcal{E}\left(f ; \mathcal{E}_{\Psi}\right)$ we get a contradiction using Theorem 7.1(b).

Theorem 7.4. Let $\Phi \in \boldsymbol{r}$ and let $g_{k}, k=1,2, \cdots$, be an increasing countable family of ideals of $\mathcal{L}$ sucb that $g_{k} \subsetneq \mathfrak{S}_{\Phi}^{0} k=1,2, \ldots$ Then $\cup_{k=1}^{\infty} g_{k} \varsubsetneqq \oint_{\phi}^{0}$.

Proof. For each $k=1,2, \cdots$, let $x^{(k)}$ be a sequence in $\delta_{\Phi}^{0}$ such that $x^{(k)} \& \Lambda\left(g_{k}\right), \Phi\left(x^{(1)}\right) \leq 1$ and $x_{n}^{(j)} \leq x_{n}^{(k)}$, for $j \leq k$, and $n=1,2, \ldots$ (the existence of the sequences $x^{(k)}, k=1,2, \cdots$, is guaranteed by the fact that the family $g_{k}, k=1,2, \ldots$, is increasing). For each $n=1,2, \ldots$ we define the positive integers $k_{n}$ and $b_{n}$ as follows: let $k_{1}$ be the first positive integer such that $\Phi\left[r_{k_{1}}\left(x^{(2)}\right)\right] \leq 1 / 2$, and let $b_{1}$ be the first positive integer such that $b_{1}>k_{1}$ and $x_{b_{1}}^{(2)} \leq x_{k_{1}+1}^{(1)}, \cdots, k_{m+1}$ be the first positive integer such that $k_{m+1} \geq b_{m}$ and $\Phi\left[r_{k_{m+1}}\left(x^{(m+2)}\right)\right] \leq 1 / 2^{m+1}$, and let $b_{m+1}$ be the first positive integer such that $b_{m+1}>k_{m+1}$ and $x_{b_{m+1}}^{(m+2)} \leq x_{k_{m}+1+1}^{(m+1)}$. Now let $x$ be the sequence in $\mathbb{R}$ defined by $x_{n}=x_{n}^{(1)}, 1 \leq n \leq k_{1} ;$ if $b_{1}>k_{1}+1$, then $x_{n}=x_{k_{1}+1}^{(1)}, k_{1}+1 \leq n<b_{1} ; x_{n}=x_{n}^{(2)}$, $b_{1} \leq n \leq k_{2} ;$ if $b_{2}>k_{2}+1$, then $x_{n}=x_{k_{2}+1}^{(2)}, k_{2}+1 \leq n<b_{2}$; etc. We see that, except for the first few terms, $x$ majorizes the sequence $x^{(k)}, k=1,2, \ldots$. Therefore $x \notin \bigcup_{k=1}^{\infty} \Lambda\left(g_{k}\right)$. In order to complete the proof of the theorem it remains to show that $x \in \mathcal{S}_{\Phi}^{f}$. Let $\epsilon$ be an arbitrary positive number and let $j$ be a positive integer such that $1 / 2^{j}<\epsilon$. Then

$$
\Phi\left[r_{k_{j}}(x)\right] \leq \sum_{i=j}^{\infty} \Phi\left(\sigma_{k_{i+1}}\left[r_{k_{i}}(x)\right]\right) \leq \sum_{i=j}^{\infty} \Phi\left(\sigma_{k_{i+1}}\left[r_{k_{i}}\left(x^{(i+1)}\right)\right]\right) \leq \sum_{i=j}^{\infty} \frac{1}{2^{i+1}}<\epsilon .
$$

Thus, $x \in \mathcal{G}_{\Phi}^{0}$ as desired.

Theorem 7.5. Let $f_{n} \in \mathbb{Q}, n=1,2, \cdots$, and let $\mathcal{J}_{n}, n=1,2, \cdots$, be a countable family of norm ideals sucb that $\mathcal{J}_{n} \varsubsetneqq \mathcal{C}, n=1,2, \ldots$. Then $V_{n=1}^{\infty} S\left(f_{n} ; g_{n}\right) \subsetneq \mathcal{C}$.

Proof. Let $g_{k}=V_{n=1}^{k} S\left(f_{n} ; \mathcal{J}_{n}\right)$. From Theorem 7.3 and [10, Theorem 2.22] it follows that $g_{k} \subsetneq \mathcal{C}, k=1,2, \ldots$. Now the statement follows from Theorem 7.4.

Corollary 7.6. Let $\mathscr{S}_{k}, k=1,2, \cdots$, be a countable family of norm ideals sucb that $g_{k} \subsetneq \mathcal{C}, k=1,2, \ldots$ Then $\bigvee_{k=1}^{\infty} g_{k} \varsubsetneqq \mathcal{C}$

The following results provide partial answers to problem $\left(a_{1}\right)$.

Theorem 7.7. Let $\Phi \in \boldsymbol{r}$ sucb that $\delta_{\Phi} \subseteq \mathcal{C}$ and for eacb $k=1,2, \ldots$ let $f_{k} \in \mathcal{Q}$ and $\Phi_{k} \in \pi$ sucb that $\mathcal{S}_{\Phi} \subsetneq \mathcal{D}\left(f_{k} ; \mathcal{S}_{\Phi_{k}}^{0}\right)$. If the family $\mathcal{D}\left(f_{k} ; \mathcal{S}_{\Phi_{k}}^{0}\right)$ is decreasing, then $\mathcal{S}_{\Phi} \subseteq \bigcap_{k=1}^{\infty} \mathscr{D}\left(f_{k} ; \delta_{\Phi_{k}}^{0}\right)$. 
Proof. For each $k=1,2, \ldots$ let $z^{(k)} \in \mathfrak{S}\left(f_{k} ; \mathfrak{G}_{\Phi_{k}}^{0}\right)$ such that $z^{(k)} \notin \mathfrak{G}_{\phi^{\bullet}}$ From Theorem 7.1(a), for each $k=1,2, \cdots$, there exists $c^{(k)} \in \Gamma$ such that $f_{k} \circ\left(c^{(k)} z^{(k)}\right) \in \circlearrowleft_{\Phi_{k}}^{0}$. Since the family $\mathfrak{X}\left(f_{k} ; \circlearrowleft_{\Phi_{k}}^{0}\right), k=1,2, \ldots$, is decreasing, by deleting the first few terms of the sequences $c^{(k)} z^{(k)}, k=1,2, \ldots$, if necessary, we can assume without loss of generality that $\Phi_{j}\left[f_{j} \circ\left(c^{(k)} z^{(k)}\right)\right] \leq 1 / 2^{k}$, $1 \leq j \leq k, k=1,2, \ldots$. For each $n=1,2, \ldots$ we define the positive integers $k_{n}$ and $b_{n}$ as follows: $k_{1}=1$; let $b_{1}$ be a positive integer such that $\Phi\left[\sigma_{b 1}\left(z^{(1)}\right)\right]$ $\geq 1$; let $k_{2}$ be the first positive integer such that $k_{2} \geq b_{1}+1, z_{k_{2}+1}^{(2)} \leq z_{b_{1}+1}^{(1)}$ and $c_{k_{2}+1}^{(2)} \geq c_{b_{1}+1}^{(1)}$; and let $b_{2}$ be a positive integer such that $\Phi\left(\sigma_{b_{2}}\left[r_{k_{2}}\left(z^{(2)}\right)\right]\right) \geq 2$; $\cdots$; having defined $k_{m}, b_{m^{\prime}} m \geq 2$, let $k_{m+1}$ be the first positive integer such that $k_{m+1} \geq k_{m}+b_{m}, z_{k_{m}+1}^{(m+1)} \leq z_{k_{m}+b_{m}}^{(m)}$, and $c_{k_{m}+1+1}^{(m+1)} \geq c_{k_{m}+b_{m}}^{(m)}$; and let $b_{m+1}$ be a positive integer such that $\Phi\left(\sigma_{b_{m+1}}\left[r_{k_{m}+1}\left(z^{(m+1)}\right)\right]\right) \geq m+1$. Now let $z \in \mathfrak{I}$ and let $c \in \Gamma$ be the sequences given by $z_{n}=z_{n}^{(1)}, c_{n}=c_{n}^{(1)}, 1 \leq n \leq b_{1}$; $z_{n}=z_{n+k_{2}-b_{1}}^{(2)}, c_{n}=c_{n+k_{2}-b_{1}}^{(2)}, b_{1}+1 \leq n \leq b_{1}+b_{2} ; \cdots$;

$$
z_{n}=z_{n+k_{m+1}-\Sigma_{j=1}^{m} b_{j}}, \quad c_{n}=c_{n+k_{m+1}}-\Sigma_{j=1}^{m} b_{j}, \quad 1+\sum_{j=1}^{m} b_{j} \leq n \leq \sum_{j=1}^{m+1} b_{j} \cdot
$$

By definition $z \notin \tilde{G}_{\Phi} ;$ in fact

$$
\lim _{k \rightarrow \infty} \Phi\left[\sigma_{k}(z)\right]=\lim _{m \rightarrow \infty} \Phi\left[\sigma_{\Sigma_{j=1}^{m} b_{j}}(z)\right] \geq \lim _{m \rightarrow \infty} \Phi\left(\sigma_{b_{m}}\left[r_{k_{m}}\left(z^{(m)}\right)\right]\right) \geq \lim _{m \rightarrow \infty} m=\infty \text {. }
$$

Finally we show that $f_{k} \delta(c z) \in \mathbb{S}_{\Phi}^{0}, k=1,2, \ldots$. To this end let $i$ be an arbitrary positive integer and let $\epsilon>0$ be given. Choose $j$ sufficiently large so that $j \geq i, 1 / 2^{j}<\epsilon / 2$. It follows that

$$
\begin{aligned}
\Phi_{i}\left(r_{k_{j}}\left[f_{i} \circ(c z)\right]\right) & \leq \sum_{m=j}^{\infty} \Phi_{i}\left[\sigma_{b_{m}}\left(r_{k_{m}}\left[f_{i} \circ\left(c^{(m)} z^{(m)}\right)\right]\right)\right] \\
& \leq \sum_{m=j}^{\infty} \Phi_{i}\left[f_{i} \circ\left(c^{(m)} z^{(m)}\right)\right] \leq \sum_{m=j}^{\infty} 1 / 2^{m}<\epsilon_{0}
\end{aligned}
$$

Since $c \in \Gamma$, it follows from Theorem 7.1(a) that $z \in \bigcap_{k=1}^{\infty} \mathscr{X}\left(f_{k} ; \Im_{\Phi_{k}}^{0}\right)$ and the proof of the theorem is complete.

Corollary 7.8. Let $\Phi \in \boldsymbol{\pi}, \mathcal{S}_{\Phi} \subseteq \mathcal{C}$ and let $\mathscr{G}_{k}, k=1,2, \ldots$, be a decreas. ing countable family of norm ideals sucb that $\delta_{\Phi} \varsubsetneqq \mathscr{G}_{k}, k=1,2, \ldots$ Then $\mathcal{S}_{\Phi} \subsetneq \bigcap_{k=1}^{\infty} g_{k}$.

Proof. Let $\Phi_{k} \in \boldsymbol{r}$ such that $\mathcal{S}_{\Phi_{k}}^{0}$ is the minimal norm ideal corresponding to $g_{k}, k=1,2, \ldots$. Since $\delta_{\Phi} \varsubsetneqq g_{k}, k=1,2, \ldots$, and the family $g_{k}, k=1,2$, $\because \cdots$, is decreasing we deduce, from Theorem 2.3 and Corollary 2.4 , that $\mathfrak{S}_{\phi} \varsubsetneqq \mathcal{S}_{\Phi_{k}}^{0}$ and $\mathcal{S}_{\Phi_{k+1}}^{0} \cong \mathcal{S}_{\Phi_{k^{\prime}}}^{0} k=1,2, \ldots$. Now the corollary follows from Theorem 7.7. 
Corollary 7.9. Let $f_{k}, k=1,2, \cdots$, be a family of convex admissible functions such that $f_{k} \chi f_{(1)}, k=1,2, \cdots$, and let $g_{k}, k=1,2, \ldots$, be a family of concave admissible functions sucb that $g_{k} \nsim f_{(1)}, k=1,2, \ldots$ Then $\bigvee_{k=1}^{\infty} S\left(g_{k} ; \mathcal{C}_{p}\right) \varsubsetneqq$ $\mathcal{C}_{p} \subseteq \bigcap_{k=1}^{\infty} \mathcal{D}\left(f_{k} ; \mathcal{C}_{p}\right)$, for every $p \geq 1$.

Proof. It can be easily checked that if $f_{j}$ are convex admissible functions and $g_{j}$ are concave admissible functions, such that $f_{j} \not f_{(1)}$ and $g_{j} \not f_{(1)}, j=1,2$, then $f_{1} \vee f_{2} \nsim f_{(1)}$ and $g_{1} \wedge g_{2} \nsim f_{(1)}$. Therefore from [10, Theorem 2.12]

$$
\bigvee_{j=1}^{k} S\left(g_{j} ; \mathcal{C}_{p}\right)=S\left(\bigwedge_{j=1}^{k} g_{j} ; \mathcal{C}_{p}\right) \varsubsetneqq \mathcal{C}_{p} \varsubsetneqq \mathscr{D}\left(\bigvee_{j=1}^{k} f_{j} ; \mathcal{C}_{p}\right)=\bigcap_{j=1}^{k} \mathcal{D}\left(f_{j} ; \mathcal{C}_{p}\right) .
$$

Thus, without loss of generality, we can assume that $\mathcal{S}\left(g_{k} ; \mathcal{C}_{p}\right), k=1,2, \ldots$, is an increasing family such that $\mathcal{S}\left(g_{k} ; \mathcal{C}_{p}\right) \varsubsetneqq \mathcal{C}_{p}, k=1,2, \ldots$, and that $\mathscr{D}\left(f_{k} ; \mathcal{C}_{p}\right), k=1,2, \cdots$, is a decreasing family such that $\mathcal{C}_{p} \varsubsetneqq \mathscr{D}\left(f_{k} ; \mathcal{C}_{p}\right), k=$ $1,2, \ldots$. Now the corollary is a consequence of Theorem 7.4 and Theorem 7.7.

The above corollary solves, in the case of the $p$-Schatten ideals, a question raised in $[10, \$ 4]$.

The following theorem is the counterpart of Corollary 7.6.

Theorem 7.10. Let $\mathscr{G}_{k}, k=1,2, \cdots$, be a countable family of norm ideals which strictly contain the trace class $\mathcal{C}_{1}$. Then $\mathcal{C}_{1} \varsubsetneqq \bigcap_{k=1}^{\infty} g_{k}$.

Proof. For each $k=1,2, \ldots$, let $\Phi_{k} \in \Re$ such that $\delta_{\Phi_{k}}^{0}$ is the minimal norm ideal corresponding to $g_{k}$. Since $\mathcal{C}_{1} \varsubsetneqq \mathcal{S}_{\Phi_{k}}, k=1,2, \cdots$, it follows from Theorem 2.3 that $\mathcal{C}_{1} \varsubsetneqq \mathcal{S}_{\Phi_{k}}^{0}, k=1,2, \ldots$. Thus it suffices to prove that $\mathcal{C}_{1} \varsubsetneqq \bigcap_{k=1}^{\infty} \mathcal{S}_{\Phi_{k}}^{0}$. To this end, we first prove that if $\Phi, \Psi \in \boldsymbol{r}$ such that $\mathcal{C}_{1} \varsubsetneqq \mathcal{S}_{\Phi}^{0}$ and $\mathcal{C}_{1} \varsubsetneqq \mathcal{C}_{\Psi}$ then $\mathcal{C}_{1} \subsetneq \mathcal{S}_{\phi}^{0} \cap \mathfrak{S}_{\Psi}^{0}$. We recall (Remark 4.7(d)) that $\delta_{\Phi}^{0} \cap \delta_{\psi}^{0}=\mathcal{S}_{\Phi}^{0} \vee$ Also, from Remark 2.5 it follows that $\inf _{k} k^{-1} \Phi\left[\sigma_{k}(u)\right]=\inf _{k} k^{-1} \Psi\left[\sigma_{k}(u)\right]=0$, where $u_{n}=1$, $n=1,2, \ldots$. We observe that if $j \leq k$, then $j^{-1} \Phi\left[\sigma_{j}(u)\right] \geq k^{-1} \Phi\left[\sigma_{k}(u)\right]$ and similarly $j^{-1} \Psi\left[\sigma_{j}(u)\right] \geq k^{-1} \Psi\left[\sigma_{k}(u)\right]$. Therefore, we deduce that $\lim _{k \rightarrow \infty} k^{-1} \Phi\left[\sigma_{k}(u)\right]$ $=\lim _{k \rightarrow \infty} k^{-1} \Psi\left[\sigma_{k}(u)\right]=0$ and hence $\lim _{k \rightarrow \infty} k^{-1}(\Phi \vee \Psi)\left[\sigma_{k}(u)\right]=0$. Again, from Remark 2.5 we conclude that $\mathcal{C}_{1} \varsubsetneqq S_{\Phi V^{*}}^{0}=\mathcal{S}_{\Phi}^{0} \cap \mathcal{S}_{\boldsymbol{\psi}}^{0}$, as desired. Now we define the sequence $\left\{\Psi_{k}\right\}$ in $\pi$ by $\Psi_{k}=\sup _{1 \leq j \leq k} \Phi_{j}, k=1,2, \ldots$. We see that $\mathcal{S}_{\Psi_{k}}^{0}$, $k=1,2, \cdots$, is a decreasing family of minimal norm ideals which are strictly larger than $\mathcal{C}_{1}$, and hence, from Corollary 7.8, we conclude that $\bigcap_{k=1}^{\infty} \delta_{\phi_{k}}^{0}=$ $\cap_{k=1}^{\infty} \mathcal{S}_{\Psi_{k}}^{0} \supsetneq \mathcal{C}_{1}$.

Our final task in the present paper is devoted to solving problem $\left(a_{2}\right)$ stated above. The following theorem implies in particular that problem $\left(a_{2}\right)$ has a negative answer.

Theorem 7.11. Let $\mathcal{G}$ be a norm ideal strictly smaller than $\mathcal{C}$. Then there exist two ideals $g_{1}$ and $g_{2}$ sucb that $g \subseteq g_{j}, j=1,2$, and $g=g_{1} \cap g_{2}$. 
Proof. Let $\Phi \in \mathfrak{r}$ such that $\mathcal{S}_{\Phi}^{0} \subseteq \mathfrak{g} \subseteq \mathfrak{S}_{\Phi}$. We shall prove that there exist two sequences $x$ and $y$ in $\mathfrak{R}$ such that $x \wedge y \in \sigma_{\phi}^{0}$. Assuming that this fact has already been proved we let $T$ and $S$ in $\mathcal{\perp}$ such that $s(T)=x$ and $s(S)=y$. It follows that the principal ideals $(T)$ and $(S)$ are not contained in $\mathscr{I}$ but $(T) \cap(S)$ $\subseteq \mathscr{g}$. Therefore letting $g_{1}=g \vee(T)$ and $g_{2}=g \vee(S)$ we conclude that $g_{1} \cap g_{2}^{\prime}=g$ but $I \varsubsetneqq \mathscr{I}_{j}^{\prime}, j=1,2$. Thus, in order to complete the proof of the theorem it suffices to find the sequences $x$ and $y$ in $\{$ with the properties mentioned above. Let $z \in G_{\Phi}^{0}$ such that $z_{n} \neq 0, n=1,2, \ldots$. Employing the fact that $\lim _{k \rightarrow \infty} \Phi\left[\sigma_{k}(u)\right]=\infty$ (because $\delta_{\Phi} \varsubsetneqq \mathcal{C}$ ), we construct by induction a sequence $\left\{k_{n}\right\}$ of positive integers as follows: let $k_{1}$ be the first positive integer such that $\Phi\left[\sigma_{k_{1}}(u)\right] \geq 1 / z_{1}$; let $k_{2}$ be the first positive integer such that $k_{2}>k_{1}$ and $\Phi_{\sigma_{k_{2}-k_{1}}}(u) \geq 2 / z_{k_{1}} ; \cdots ;$ let $k_{n+1}$ be the first positive integer such that $k_{n+1}>k_{n}$ and

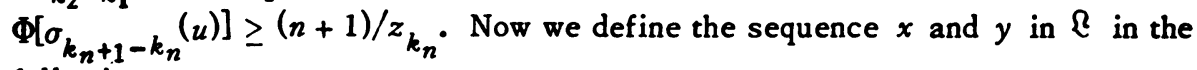
following way:

$$
\begin{array}{lll}
x_{n}=z_{1}, & y_{n}=z_{n}, & 1 \leq n \leq k_{1} ; \\
x_{n}=z_{n}, & y_{n}=z_{k_{1}}, & k_{1}+1 \leq n \leq k_{2} ; \\
x_{n}=z_{k_{2}}, & y_{n}=z_{n}, & k_{2}+1 \leq n \leq k_{3} ; \\
x_{n}=z_{n}, & y_{n}=z_{k_{3}}, & k_{3}+1 \leq n \leq k_{4} ; \cdots
\end{array}
$$

It readily follows that $x \wedge y=z$ and

$$
\lim _{k \rightarrow \infty} \Phi\left[\sigma_{k}(x)\right]=\lim _{n \rightarrow \infty} \Phi\left[\sigma_{k 2 n}(x)\right] \geq \lim _{n \rightarrow \infty} z_{k} \Phi\left[\sigma_{k_{2 n}}(u)\right]=\infty
$$

and

$$
\lim _{k \rightarrow \infty} \Phi\left[\sigma_{k}(y)\right]=\lim _{n \rightarrow \infty} \Phi\left[\sigma_{k_{2 n+1}}(y)\right] \geq \lim _{n \rightarrow \infty} z_{k_{2 n+1}} \Phi\left[\sigma_{k_{2 n+1}}(u)\right]=\infty .
$$

Therefore, $x \wedge y \in \mathcal{E}_{\Phi}^{0}$, but $x \notin \mathcal{E}_{\phi}, y \notin \mathcal{E}_{\Phi}$ and our claim is established.

\section{REFERENCES}

1. A. Brown, C. Pearcy and N. Salinas, Ideals of compact operators on Hilbert space, Michigan Math. J. 18 (1971), 373-384. MR 45 \#909.

2. J. W. Calkin, Two-sided ideals and congruences in the ring of bounded operators in Hilbert space, Ann. of Math. (2) 42 (1941), 839-873. MR 3, 208.

3. K. Fan, Maximum properties and inequalities for the eigenvalues of completely continuous operators, Proc. Nat. Acad. Sci. U.S.A. 37 (1951), 760-766. MR 13, 661.

4. D. J. H. Garling, On ideals of operators in Hilbert space, Proc. London Math. Soc. (3) 17 (1967), 115-138. MR 34 \#8208.

5. I. C. Gohberg and M. G. Kreĭn, Introduction to the theory of linear nonselfadjoint operators in Hilbert space, "Nauka", Moscow, 1965; English transl., Transl. Math. Monographs, vol. 18, Amer. Math. Soc., Providence, R. I., 1969. MR 36 \#3137; 39 \#447. 
6. 1. C. Gohberg and M. G. Krein, On the theory of triangular representations of nonselfadjoint operators, Dokl. Akad. Nauk SSSR 137 (1961), 1034-1037 = Soviet Math. Dokl. 2 (1961), 392-395. MR 25 \#3370.

7. A. Horn, On the singular values of a product of completely continuous operators, Proc. Nat. Acad. Sci. U.S.A. 36 (1950), 374-375. MR 13, 565.

8. B. S. Mitjagin, Normed ideals of intermediate type, Izv. Akad. Nauk SSSR Ser. Mat. 28 (1964), 819-832; English transl., Amer. Math. Soc. Transl. (2) 63 (1967), 180-194. MR 30 \#4142.

9. B. S. Mitjagin and A. S. Svarc, Functors in categories of Banach spaces, Uspehi Mat. Nauk 19 (1964), no. 2 (116), 65-130 = Russian Math. Surverys 19 (1964), no. 2, 65127. MR 29 \#3866.

10. N. Salinas, Ideal sets and ideals of compact operators on Hilbert space, Indiana Univ. Math. J. 22 (1972), 505-521.

11. - Ideals of commutators of compact operators, Acta Sci. Math.. (Szeged) (to appear).

12. R. Schatten, Norm ideals of completely continuous operators, Ergebnisse der Math. und ihrer Grenzgebiete, N. F., Heft 27, Springer-Verlag, Berlin, 1960. MR 22 \#9878.

13. H. Weyl, Inequalities between the two kinds of eigenvalues of a linear transformation, Proc. Nat. Acad. Sci. U.S.A. 35 (1949), 408-411. MR 11, 37.

14. A. Zaanen, Linear analysis. Measure and integral, Banach and Hilbert space, linear integral equations, Interscience, New York; North-Holland, Amsterdam; Noordhoff, Groningen, 1953. MR 15, 878.

SCHOOL OF MATHEMATICS, THE INSTITUTE FOR ADVANCED STUDY, PRINCETON, NEW JERSEY 08540

Current address: Department of Mathematics, University of Kansas, Lawrence, Kansas 66044 